\title{
Neue Ungleichheitsfragen, neue Cleavages? Ein internationaler Vergleich der Einstellungen in vier Ungleichheitsfeldern
}

\author{
Thomas Lux $\cdot$ Steffen Mau $\cdot$ Aljoscha Jacobi
}

Angenommen: 14. Oktober 2021 / Online publiziert: 11. November 2021

(C) Der/die Autor(en) 2021

Zusammenfassung Der Beitrag fragt vor dem Hintergrund neuer gesellschaftlicher Konfliktthemen nach der sozialstrukturellen Fundierung von Ungleichheitseinstellungen. Es werden vier Ungleichheitsachsen in den Blick genommen: (1) ObenUnten-Ungleichheiten, bei denen die ökonomische Ressourcenverteilung im Mittelpunkt steht, (2) Innen-Außen-Ungleichheiten, die sich auf territorialen Zugang, Migration und Mitgliedschaft beziehen, (3) Wir-Sie-Ungleichheiten, die die gesellschaftliche Anerkennung von Diversität umfassen, und (4) Heute-Morgen-Ungleichheiten, die sich auf Fragen der Generationengerechtigkeit und ökologischen Nachhaltigkeit richten. In jedem Bereich werden die Ausprägungen und Strukturierungen der sozialen Orientierungen aufgezeigt und in der Literatur gängige Erwartungen hierzu getestet. Zentral geht es um die Frage, ob die Einstellungsmuster in einer Weise mit sozialstrukturellen Differenzierungen einhergehen, dass man von Cleavages sprechen könnte. Auf der Basis des European Social Survey aus dem Jahr 2016 werden die Einstellungsstrukturen deskriptiv sowie faktoren- und regressionsanalytisch für eine ausgewählte Gruppe europäischer Länder (Schweden, Frankreich, Italien, Deutschland, Polen und Ungarn) untersucht. Der Kernbefund ist, dass sich, erstens, kein zweidimensionaler Einstellungsraum zeigt, bei dem die alten ökonomischen Ungleichheiten den neuen Ungleichheiten gegenüberstehen, und, zweitens, nur punktuell und nicht in allen untersuchten Ländern starke Cleavages zu finden sind. Diese zeigen sich am ehesten beim Migrationsthema.

\footnotetext{
Thomas Lux $(\bowtie) \cdot$ Steffen Mau $\cdot$ Aljoscha Jacobi Institut für Sozialwissenschaften, Lehrbereich Makrosoziologie, Humboldt-Universität zu Berlin, Unter den Linden 6, 10099 Berlin, Deutschland

E-Mail: thomas.lux@hu-berlin.de

Steffen Mau

E-Mail: steffen.mau@hu-berlin.de

Aljoscha Jacobi

E-Mail: jacobalj@hu-berlin.de
} 
Schlüsselwörter Spaltungslinien · Neue Ungleichheiten · Konflikt · Polarisierung • Einstellungen

\title{
New inequalities, new cleavages? An international comparison of attitudes in four fields of inequality
}

\begin{abstract}
The article investigates the socio-structural foundations of attitudes toward inequality in light of new issues of social conflict. Four axes of inequality are taken into account: (1) top-bottom inequalities, which focus on economic resource distributions, (2) inside-outside inequalities, which relate to territorial access, migration, and membership, (3) us-them inequalities, which encompass social recognition of diversity, and (4) today-tomorrow inequalities, which focus on issues of intergenerational equity and environmental sustainability. The article quantitatively assesses the cleavage structure in each area and tests expectations on relationships found in the literature. The central question is: are attitudes polarized and associated with sociostructural characteristics in such a way that one could speak of cleavages? Based on the European Social Survey from 2016, the attitudinal structures are examined descriptively as well as with factor and regression analyses for a selected group of European countries (Sweden, France, Italy, Germany, Poland, and Hungary). The key finding is, first, that no two-dimensional distribution of attitudes is evident in which the old, economic inequalities are simply opposed to the new inequalities; and, second, that strong cleavages can be found only in some countries on some issues. The most consistent socio-structural cleavage forms around the topic of migration.
\end{abstract}

Keywords Cleavage structure · New inequalities · Conflict · Polarization · Attitudes

\section{Nouvelles inégalités, nouveaux clivages? Une comparaison internationale des attitudes face aux inégalités dans quatre domaines}

Résumé Cet article s'interroge sur les soubassements sociostructurels des attitudes face aux inégalités dans le contexte de l'émergence de nouveaux thèmes de conflit dans la société. Les inégalités sont examinées dans quatre dimensions : (1) les inégalités haut-bas centrées sur la répartition des ressources économiques, (2) les inégalités intérieur-extérieur concernant l'accès au territoire, les migrations et l'appartenance, (3) les inégalités nous-eux englobant la reconnaissance sociale de la diversité et (4) les inégalités aujourd'hui-demain portant sur les questions de justice intergénérationnelle et de durabilité écologique. Dans chaque dimension, les formes et la structuration des orientations sociales sont mises en évidence et des hypothèses courantes dans la littérature sur le sujet sont testées. La question centrale est de savoir si les différences d'attitudes coïncident avec les différenciations sociostructurelles de telle sorte qu'ont puisse parler de clivages. Sur la base de l'Enquête sociale européenne (ESS) de 2016, la structure des attitudes dans un groupe de pays européens sélectionnés (Suède, France, Italie, Allemagne, Pologne, Hongrie) est décrite et soumise à une analyse factorielle et de régression. Le principal résultat de cette étude est, premièrement, qu'aucun espace des attitudes bidimensionnel 
n'apparaît dans lequel les anciennes inégalités économiques s'opposeraient aux nouvelles inégalités et, deuxièmement, qu'on observe des clivages prononcés seulement de manière ponctuelle et dans certains des pays étudiés. Ces clivages sont le plus susceptibles de se manifester sur la question des migrations.

Mots-clés Lignes de scission · Nouvelles inégalités · Conflit · Polarisation · Attitudes

\section{Einleitung}

Die Cleavage-Theorie der Politikwissenschaft (Lipset und Rokkan 1967) definiert Spaltungslinien als langfristige Zuordnungen von Wählerschaften zu politischen Lagern, die mit strukturellen Fraktionierungen innerhalb der Bevölkerung in Verbindung stehen. Für diese strukturellen Fraktionierungen können ökonomische Interessen, Religion, kulturelle Prägungen oder regionale Disparitäten eine Rolle spielen. Im Vergleich zur Politikwissenschaft ist eine soziologische Perspektive weniger an der Lagerbildung der Elektorate interessiert, sondern primär am Zusammenhang zwischen sozialstrukturellen Positionen und sozialen und politischen Orientierungen. Sie schaut gewissermaßen auf den Unterbau, auf dem politische Wählermobilisierung aufsitzen kann. Mit dieser konzeptionellen Vorstellung gesellschaftlich salienter Spaltungslinien im Hinterkopf geht es in diesem Aufsatz um den Zusammenhang zwischen Sozialstruktur und Einstellungen zu gesellschaftlichen Ungleichheiten.

Die Frage danach, was die Gesellschaft zusammenhält und welches die wesentlichen Fliehkräfte sind, kann als die Gretchenfrage des Fachs verstanden werden, die aber immer nur vorläufig und partiell beantwortet werden kann. Dass dabei die Ungleichheitsfrage eine zentrale Rolle spielt, liegt auf der Hand, entscheidet sie doch über soziale Nähe und Distanz unterschiedlicher Bevölkerungsgruppen, über Formen der Anerkennung und Einbeziehung und über Erfahrungen von Exklusion und Inklusion. Oft stehen bei öffentlichen Diskussionen die Verteilungen der Einkommen und Vermögen im Vordergrund, aber die gesellschaftlichen Ungleichheitsfragen und die damit verbundenen Konflikte sind vielfältiger. Spätestens seit den Diskussionen um sogenannte horizontale Disparitäten, Intersektionalität, Teilhabe oder die Generationengerechtigkeit ist bekannt, dass das Thema der Ungleichheit mit einem Fokus auf vertikale sozioökonomische Ungleichheiten kaum hinreichend zu erfassen ist. Diese pluralisierten Ungleichheiten sind keinesfalls neu, aber sie werden heute anders artikuliert und finden auf andere Weise in den öffentlichen Diskurs.

Wir möchten mit unserem Aufsatz dieser Vervielfältigung der Perspektiven entsprechen, allerdings ohne die Frage nach der sozialstrukturellen Fundierung der Ungleichheitsthematik aufzugeben. Wir greifen dafür auf ein Raster zurück, das wir in einem früheren Aufsatz (Mau et al. 2020) entwickelt und angewandt haben, und erweitern es sowohl inhaltlich als auch empirisch. Wir nehmen vier Achsen potenzieller Ungleichheitskonflikte in den Fokus, die zwar jeweils in der Literatur eingeführt, aber weder begrifflich in einen gemeinsamen Rahmen gestellt noch im Zusammenhang analysiert wurden. Dies sind klassische vertikale Ungleichheiten (Oben-Unten-Ungleichheiten), Ungleichheiten der Mitgliedschaft und der Mi- 
gration (Innen-Außen-Ungleichheiten), Anerkennungsungleichheiten (Wir-Sie-Ungleichheiten) und Fragen der Generationengerechtigkeit und der ökologischen Nachhaltigkeit (Heute-Morgen-Ungleichheiten). An diesen Ungleichheitsachsen interessiert uns jeweils, wie sie sich in den Einstellungen unterschiedlicher Statusgruppen widerspiegeln, kurz: ob sie sich als sozialstrukturell bedeutsame Spaltungslinien darstellen. Einzelne empirische Befunde zu dieser Frage finden sich zwar, aber sie sind stark portioniert und oftmals unverbunden. Im vorliegenden Aufsatz wollen wir uns dem Themenfeld umfassend und systematisch nähern: Erstens untersuchen wir die genannten Ungleichheitsachsen in einem Zusammenhang (mit einem Datensatz und einer einheitlichen Untersuchungsanlage). Zweitens prüfen wir den Zusammenhang zwischen den Einstellungen und einer ganzen Reihe sozialstruktureller Faktoren, wobei wir über rein vertikale Aspekte hinausgehen und auch horizontale Merkmale berücksichtigen. Drittens unternehmen wir einen internationalen Vergleich mit dem Ziel herauszufinden, ob es nun einen Unterschied macht, über welche Gesellschaft man redet. Sind also die möglicherweise auffindbaren Spaltungslinien in den Einstellungen länderübergreifend ähnlich konturiert oder gibt es nationale Spezifika?

Unser Vorhaben gliedert sich in Versuche ein, ein Lagerungsbild ,jenseits von Kapital und Arbeit" zu kartieren und so die neueren Themen und Agenden der gesellschaftlichen Politisierung sozialer Ungleichheit zu erfassen. Seien es identitätspolitische Ankerkennungskämpfe, die gern und oft beschworene „Migrationskrise" oder die Fridays-for-Future-Bewegung: Sie alle haben Themen auf die Agenda gebracht, von denen wir nur oberflächlich wissen, wie sie in der allgemeinen Bevölkerung verstanden und unterstützt werden und, ebenso entscheidend, ob sie eine sozialstrukturelle Verankerung haben oder davon entkoppelt sind. Im letzteren Fall könnte man wohl von aktuellen politischen Konflikten, aber nicht von längerfristigen sozialstrukturellen und sozio-politischen Spaltungslinien (Cleavages) sprechen.

\section{Neue Konfliktfelder in der Diskussion}

In der Diskussion zu neuen Spaltungslinien lassen sich mehrere Zugänge identifizieren, die zwar mit eigenen, aber doch inhaltlich verbundenen Begrifflichkeiten operieren. Ausgangspunkt ist jeweils die Annahme, dass die politische Rechts-LinksOrdnung, die sich aus der Grundspannung divergierender Interessen von Kapital und Arbeit speist, angereichert bzw. überlagert wird. Das Vordringen postmaterieller Werte in der Gesellschaft, die Öffnung des nationalgesellschaftlichen Containerraums, die Veränderung von Bildungs- und Berufsstrukturen, die Mediatisierung der Gesellschaft und die politische Polarisierung werden als Antriebskräfte der Veränderung des Konfliktraumes genannt (Ford und Jennings 2020). Die Aushandlung von Gleichheit spielt sich nicht mehr nur auf dem ökonomischen Feld ab, sondern hat sich vervielfältigt und betrifft auch kulturelle und soziale Fragen von Anerkennung, Teilhabe und Chancengleichheit (Foroutan 2019).

Im Mittelpunkt einer Vielzahl von politikwissenschaftlichen Arbeiten steht die Frage nach der Restrukturierung des (partei-)politischen Konfliktraums. Beobachtet wurde beispielsweise das Aufkommen ethnonationalistischer und rechter Parteien am einen Ende des Parteienspektrums und eher postmateriell orientierter, auf Diver- 
sität, Individualrechte und Ökologie setzende Parteien am anderen Ende. Marks et al. (2002) fassen diese Spaltung mit den Begriffen TAN (für Traditional/Authoritarian/ Nationalist) und GAL (für Green/Alternative/Libertarian). Darauf aufbauend ist eine Reihe von Arbeiten erschienen, die noch dezidierter auf die Wirkungen von Liberalisierung, europäischer Integration und Migration auf die Aufstellung unterschiedlicher Parteienfamilien im europäischen Kontext blickt (Kriesi et al. 2008, 2012). Diese Arbeiten betonen, dass die wirtschaftlichen und kulturellen Veränderungen Gewinner und Verlierer produzieren, weil Gruppen unterschiedlich gewappnet seien, sich gegen neue Konkurrenzen zu behaupten oder kulturelle Geltungsansprüche durchzusetzen - es käme zu einer Neustrukturierung gesellschaftlicher Konflikte, die sich auch in der Parteienlandschaft und im Wählerverhalten niederschlüge (Kriesi und Grande 2004). Mit Blick auf die Wählerschaften gelten jene mit niedrigem Human- und Bildungskapital nicht nur als Befürworter eines umverteilenden und sozial schützenden Staates, sondern auch als Unterstützer „,demarkationistischer“ und auf nationale Schließung setzender politischer Angebote, während Gruppen mit einer besseren Ressourcenausstattung und höherer Bildung die „Gewinner“ seien und sich daher eher in Richtung von Parteien orientieren, die für Liberalisierung und kulturelle Offenheit stehen. In der Literatur gibt es eine Diskussion dazu, ob es eher kulturelle denn ökonomische Gründe sind, die vor allem die unteren Schichten dazu bringen, rechte Parteien zu wählen, und ob wir ein ,class voting“ - verstanden als systematischer Nexus zwischen dem sozioökonomischen Standort und der Parteipräferenz von Wählern - in veränderter Form beobachten können (Rydgren 2013). Ganz grundlegend wird von einem Bedeutungszuwachs der sozio-kulturellen Konfliktlinie gegenüber materiellen Verteilungsfragen im politischen Wettbewerb ausgegangen (Bornschier 2010).

Die neuere Diskussion zu Kosmopolitismus versus Kommunitarismus, die explizit die Frage von Öffnung und Schließung des Nationalstaates in den Mittelpunkt stellt und eine neue Konfliktlinie behauptet, schließt hier an. Auch hier gilt die Unterscheidung zwischen Globalisierungsgewinnern und Globalisierungsverlierern als maßgeblich (de Wilde et al. 2019; Zürn und de Wilde 2016). Darüber hinaus wird ein Zusammenhang zwischen der sozialstrukturellen Position und einer spezifischen „Globalisierungskompetenz“ hergestellt, die auf ein kosmopolitisches „Oben“ und ein kommunitaristisches „Unten“ verweist: „Auf der einen Seite sammeln sich die Kosmopoliten als Grenzöffner und Vertreter universeller Menschenrechte. Kosmopoliten sind die Globalisierungsgewinner, besser gebildet und ausgestattet mit mobilem Human-, Sozial- und Kulturkapital. Auf der anderen Seite stehen als tendenzielle Globalisierungsverlierer die Kommunitaristen mit vergleichsweise niedriger Bildung, geringerem Einkommen und lokal-stationärem Human-, Sozial- wie Kulturkapital. [...] Die ökonomische und kulturelle Konfliktdimension überlappen sich in hohem Maße.“ (Merkel 2017a, S. 9) Wiederum gilt: Diese „Globalisierungsverlierer" seien, anders als die Gruppen mit besserem Humankapital, stark von der Schutzfunktion des nationalen Wohlfahrtsstaates abhängig, was sie zu Unterstützern einer umverteilenden, aber doch national exklusiven Sozialpolitik machen sollte. Auch wenn darauf hingewiesen wird, dass diese Beschreibungen zunächst auf Reinformen oder Idealtypen zielen, wird zugleich dargelegt, dass sich reale und empirisch vorfindbare Cleavages entlang der Kosmopolitismus-Kommunitarismus-Dimension 
finden lassen sollten, wofür die Rede von Polarisierung, Konflikten, Trennlinien oder einem kulturellen Graben zwischen den Gruppen spricht, die letztlich auf spezifische Ausprägungen von sozialen und politischen Orientierungen innerhalb der Bevölkerung verweist (Merkel 2021).

Diese Perspektiven legen nahe, dass sich neue gesellschaftliche Konfliktfelder und „Lager“ mit spezifischen Einstellungsbündeln herausgebildet haben, die auch über die Sozialstruktur hinweg unterschiedlich stark ausgeprägt sind. Schon der Begriff der Konfliktlinie impliziert, dass es keine einzelnen (und isolierten) normativen oder politischen Positionen sind, um die es geht, sondern Einstellungssyndrome, also Bündel zusammengehörender oder zusammen auftretender Einstellungen, die auf zwei Lager hinweisen. In etlichen publizistischen Beiträgen werden so unterschiedliche Dinge wie Migration, Anerkennung von Homosexualität, kultureller Globalismus und Nachhaltigkeit zu einem Einstellungskomplex erklärt, der dann wahlweise das Etikett „,sozio-kultureller Liberalismus“ oder „liberaler Kosmopolitismus“ erhält (Heisterhagen 2018; Lilla 2018; viele solche Einordnungen finden sich auch bei Koppetsch 2019). Wolfgang Merkel (2017b, S. 53) schreibt etwa: „Kosmopoliten priorisieren individuelle Rechte, offene Grenzen, liberale Zuwanderung, erleichterte Einbürgerung, kulturellen Pluralismus sowie eine globale Verantwortung für universell gültige Menschenrechte und den Umweltschutz." Demgegenüber seien die kommunitaristischen Gruppen eher traditionell in ihrer Sicht auf Geschlechtergerechtigkeit und sexuelle Diversität, präferierten ethnische Homogenität und nationalgesellschaftliche Schließung gegenüber Offenheit und Multikulturalität und seien zurückhaltender bei der ökologischen Frage, während sie starke sozialpolitische Umverteilungsinteressen hätten. Entsprechend wird die These vertreten, dass es vor allem ,die da oben“ seien, die sich für gleichgeschlechtliche Ehen, Gender-Mainstreaming, Ökologie und Zuwanderung einsetzten, womit sie sich von den materiellen Problemen derer ,da unten“ zunehmend entfernen würden (ebd., S. 56). ${ }^{1}$

In der deutschen Diskussion haben die Arbeiten von Andreas Reckwitz (2017, 2019) die These popularisiert, dass die Postindustrialisierung, die Bildungsexpansion und der kulturelle Wertewandel vor allem in der gesellschaftlichen Mittelschicht Veränderungen herbeigeführt haben. Statt die „Mittelschicht“ als einheitlich zu begreifen, teilt er sie in eine neue Mittelklasse - geprägt von Selbstverwirklichung, Offenheit und urbanem Kosmopolitismus - und eine alte Mittelklasse, die kulturell in der Defensive ist, zudem immobil und auf den alten nationalgesellschaftlichen Ordnungsrahmen fixiert sei. Dieser Analyse zufolge wären die ,apertistischen“ Haltungen des politischen Kosmopolitismus, der Toleranz und des ökologischen Bewusstseins das Programm der neuen (kulturellen) Mittelklasse, während ,demarkationistische“ Haltungen, das Festhalten am Alten und die Abwehr von Diversität und Offenheit als das Programm einer sich (kulturell wie ökonomisch) verteidigenden

\footnotetext{
${ }^{1}$ In diesem Zusammenhang wird auch diskutiert, ob die Sozialdemokratie nicht zunehmend zwischen einer auf Umverteilung setzenden „Klassenpolitik“ und einer stärker kulturliberal ausgerichteten „Identitätspolitik“ eingeklemmt sei. Ein Vorwurf lautet, mit der Ausrichtung auf Identitätspolitik seien die ,klassischen“ Ungleichheitsfragen vernachlässigt worden (Fukuyama 2019; Heisterhagen 2018). Nancy Fraser (2017) spricht sogar vom ,progressiven Neoliberalismus“, in dem sich Antirassismus, Multikulturalismus, die Anerkennung nicht-heteronormativer Lebensformen und ökologische Orientierungen mit einer stärker liberalen und wenig auf Umverteilung setzenden Haltung verbinden.
} 
alten Mittelklasse gelten. Bei den sozial und kulturell besser gestellten Mittelschichten finden sich auch moralisch aufgeladene Formen der ,ökologischen Distinktion“, durch die soziale Grenzziehungen praktiziert werden (Neckel 2018).

In der empirischen Analyse haben wir bislang allenfalls Mosaiksteine, die diese Zusammenhänge erhellen. Norris und Inglehart (2019) vertreten die These eines kulturellen Backlashes gegen den langfristigen liberalen Wertewandel. Sie sehen Einstellungen zu Geschlecht, Homosexualität, Migration und Globalisierung als mehr oder weniger zusammenhängenden Komplex (ebd., S. 34), berücksichtigen aber nicht die sozialpolitischen Einstellungen. Die Liberalisierung sei vor allem vom Generationenwechsel angetrieben, der Backlash die Reaktion der konservativen Gruppierungen auf diesen Wertewandel. Ökonomische Entwicklungen spielten bei alledem nur eine untergeordnete katalysierende Rolle (ebd., S. 133). Die Literatur zu den Auswirkungen des technologischen Wandels stellt dagegen eine direkte Verbindung zwischen bedrohten sozialstrukturellen Positionen und der Forderung nach Umverteilung her (Rueda und Thewissen 2018). Kurer und Palier (2019) generalisieren diesen Zusammenhang: Der Strukturwandel führe zum Verlangen nach kulturellem, sozialem und ökonomischem Protektionismus. Gidron und Hall (2017) betonen in diesem Kontext nicht die direkten materiellen Effekte, sondern den Verlust an subjektivem sozialen Status. Hier ist die Vermutung, dass bedrohte Positionen zu ,demarkationistischen“ Einstellungen führen. Oesch und Rennwald (2018) differenzieren acht sozioökonomische Berufsklassen, die nicht nur die hierarchische Schichtung erfassen, sondern auch horizontale Differenzen zwischen unterschiedlichen Arbeitslogiken. Den politikwissenschaftlichen Analysen sehr ähnlich kartieren sie diese Klassen und die politischen Parteien in ihrem Verhältnis zueinander und beziehen sie auf eine ökonomische Achse (sozioökonomische Ungleichheit und Redistribution) und eine kulturelle Achse (mit den Polen libertär und traditionell). Dabei zeigt sich beispielsweise, dass Angehörige der soziokulturellen Professionen ökonomisch links und kulturell libertär ausgerichtet sind, die kleinen Handwerker und Gewerbetreibenden hingegen eher marktorientiert und kulturell traditionell, die Arbeiterschicht wiederum umverteilungsaffin und kulturell eher weniger offen und anerkennungsbereit. Andere Analysen zeigen, dass die Arbeiterschicht und die einfachen Beschäftigten im Dienstleistungsbereich einer ökonomischen Umverteilung eher positiv gegenüberstehen und negativer als höhere Statusgruppen auf Migrationsfragen blicken, während es bei den soziokulturellen Professionen umgekehrt ist (Lindh und McCall 2020). Die Autoren weisen dabei darauf hin, dass Klasseninteressen und Klassenidentitäten nicht nur an ökonomischen Fragen hängen, sondern zunehmend andere Ungleichheiten (etwa zwischen ethnischen Gruppen, hinsichtlich sexueller Identität etc.) die politischen Orientierungen beeinflussen (ebd., S. 433).

Dieser Spur folgend wollen wir in diesem Aufsatz das Verhältnis zwischen sozialstrukturellen Positionen und Einstellungsmustern genauer untersuchen, wobei bei uns Ungleichheitseinstellungen im Zentrum stehen. Statt von vornherein eine sozioökonomische und eine soziokulturelle Dimension zu unterscheiden, wollen wir das Einstellungsset weiter ausdifferenzieren. Wir verwenden eine von uns schon zuvor genutzte Heuristik zu unterschiedlichen Ungleichheitsachsen und erweitern diese. Man kann begründet vermuten, dass das In-einen-Topf-Werfen unterschiedlicher Einstellungsaspekte zu Fehlschlüssen führen kann, wenn sich die Einstellungsprofi- 
le als weniger kohärent erweisen sollten (vgl. auch Biskamp 2020, der empirische Hinweise dazu gibt). Das wäre nämlich der Fall, wenn das unterstellte oder abgeleitete Gesamtsyndrom in einzelne Dimensionen mit sehr unterschiedlichen Präferenzen und Einstellungsmustern aufgespalten werden könnte (oder sogar müsste). Ebenso finden sich inhaltliche Argumente dafür, einer zu schroffen Entgegensetzung von Interessenpolitik und Identitätspolitik skeptisch gegenüberzutreten, da die Forderungen nach materieller Gerechtigkeit oft auch eine spezifisch kulturelle Komponente besitzen; umgekehrt sind viele Anerkennungskämpfe auch materielle Kämpfe (Fraser und Honneth 2003; van Dyk 2019). Aus anderen Forschungen ist zudem bekannt, dass man bei Einstellungsprofilen nicht immer hohe Kohärenzerwartungen haben sollte und diese in sich widersprüchlich sein können (siehe beispielsweise die SplitConsciousness-These von Kluegel und Smith 1986; Arts und Gijsberts 1998).

Aufbauend auf bisherigen Arbeiten (Mau et al. 2020) unterscheiden wir vier Typen von Ungleichheiten als Bewertungsgegenstände, die wir als Oben-Unten-, Innen-Außen-, Wir-Sie- und Heute-Morgen-Ungleichheiten bezeichnen. Was meint das jeweils?

Oben-Unten-Ungleichheiten beschreiben die sozioökonomische Dimension, die auf der traditionellen Unterscheidung zwischen Kapital und Arbeit aufsitzt und anhand von Einstellungen zu ökonomischer Ungleichheit und staatlicher Redistribution abgebildet wird.

Innen-Außen-Ungleichheiten erfassen den „Kampf über Grenzen“ (Zürn 2020, S. 165) im Hinblick auf Fragen der Migration. Es geht beispielsweise um territorialen Zugang und Inklusion in soziale Sicherungssysteme, etwa um die Frage, ob neue Gruppen hinzukommen und mitgliedschaftliche Rechte einfordern dürfen, oder um die Frage, ob Migration als Bereicherung empfunden oder abgelehnt wird.

Wir-Sie-Ungleichheiten drehen sich um die Anerkennung und rechtliche Gleichstellung von nicht-heteronormativen Lebensformen, diversen Identitäten und Minoritäten, also um Aspekte, die oft unter dem analytisch ungenauen und stumpfen Begriff der ,Identitätspolitik“ versammelt werden. Hier geht es um Diskriminierungserfahrungen, Anerkennungsbestrebungen und Autonomieansprüche sozialer Gruppen, die in einer Gesellschaft leben (das unterscheidet diese Ungleichheiten zumindest analytisch von den Innen-Außen-Ungleichheiten ${ }^{2}$ ).

Heute-Morgen-Ungleichheiten zielen auf das Feld von Ökologie und Nachhaltigkeit, das ebenfalls ein Feld der Ungleichheit ist, da es auch um ungleich verteilte Umweltrisiken, die Ressourcenfrage und das Verhältnis der Generationen geht.

Nimmt man diese vier Ungleichheitsachsen in den Blick, dann erhält man ein Panorama medial und diskursiv stark aufgeladener Themen, für die es zu fragen gilt, ob sie tatsächlich als gesellschaftliche Spaltungsstrukturen beschrieben werden können. Sie dienen uns als Leitheuristik zur Disaggregation der oft auf Einstellungssyndrome abhebenden „Lagertheorien“. Empirisch gibt es für den deutschen Fall bereits Hinweise, dass die ersten drei der genannten Ungleichheitsachsen als eigenständige Dimensionen verstanden werden können, die auch unterschiedliche Antwortmuster über die Bevölkerung hinweg aufzeigen (Mau et al. 2020); für die

\footnotetext{
2 Es gibt aber auch in der Art der Politisierung im öffentlichen Diskurs Überlappungen mit den Wir-SieUngleichheiten, etwa bei der Frage des Migrationshintergrundes oder der ethnischen Identität.
} 
vierte Achse wäre dies noch zu prüfen. Ein zweiter wesentlicher Mehrwert unseres Beitrags besteht im internationalen Vergleich. Die Forschung konzentriert sich zumeist auf die ,westliche Welt“ (vor allem Westeuropa und die USA). Mit Italien, Frankreich, Deutschland und Schweden nehmen wir ebenfalls ein Set westlicher Länder in den Blick. Darüber hinaus beziehen wir mit Polen und Ungarn zwei osteuropäische Länder ${ }^{3}$ in unser Sample ein, um mögliche Spezifika aufzudecken.

Die Aufbereitung der Literatur zu den Ungleichheitsachsen und ihrer sozialstrukturellen Fundierung kann an dieser Stelle nur summarisch und ohne Anspruch auf Vollständigkeit erfolgen.

Die Erforschung der Einstellungen zu Oben-Unten-Ungleichheiten ist gewissermaßen der Klassiker der Einstellungsforschung mit einer entsprechend langen Tradition (z. B. Svallfors 1997, 2012; Mau 2003; Lux 2011). Standardbefund ist hier, dass die Ungleichheitskritik wie auch die Präferenz für staatliche Umverteilung einen sozialen Gradienten besitzen: Mit höherem Sozialstatus schwächt sich die Ungleichheitskritik ab, und es sinkt die Unterstützungsbereitschaft für umverteilende staatliche Maßnahmen. Die stärksten Unterstützer redistributiver Politik sind die unteren Schichten und die Arbeiterklasse (z. B. Lindh und McCall 2020). Im globalen Ländervergleich werden eine hohe zeitliche Stabilität der Einstellungen innerhalb der Länder sowie ein starker Zusammenhang mit dem Ausmaß der faktischen Umverteilung festgestellt - Bürgerinnen und Bürger in Ländern mit starker Umverteilung stimmen Umverteilung eher zu (Brooks und Manza 2008). Innerhalb Europas besteht dieser Zusammenhang allerdings nur bedingt. Postsozialistische Länder mit niedriger Umverteilung von Einkommen erzielen in Umfragen besonders hohe Zustimmungswerte zu Umverteilung bei niedrigerem Vertrauen in die bestehenden Institutionen (Roosma et al. 2013; Svallfors 2012). Der soziale Gradient in der Zustimmung zu umverteilenden Maßnahmen ist insbesondere in skandinavischen Ländern stark ausgeprägt, deutlich schwächer ist er hingegen in Südeuropa (Evans und Kelley 2018). In postsozialistischen Ländern lassen sich insgesamt ebenfalls vergleichsweise starke soziale Gradienten erkennen, allerdings bestehen hier recht große Unterschiede zwischen den Ländern (Pittau et al. 2013).

Die Innen-Außen-Ungleichheiten werden vor allem über die Einstellungen zu Migration und zur Inklusion von Zuwanderergruppen erfasst. Aus sozialstruktureller Perspektive sind es hier die oberen Statusgruppen (Bildung, Einkommen etc.), die eine größere Offenheit und Inklusionsbereitschaft aufweisen. Die unteren Schichten sind migrationsskeptischer und weisen stärker wohlfahrtschauvinistische Präferenzen auf (Lindh und McCall 2020; Mau und Mewes 2012; van der Waal et al. 2010). Die „Education as liberation“-These geht davon aus, dass vor allem Personen mit höherer Bildung aufgrund kultureller und ökonomischer Faktoren positivere Einstellungen gegenüber Migrationsgruppen haben und eine stärkere Inklusionsbereitschaft aufweisen (Eick 2020). Im Ländervergleich finden Studien auch innerhalb Europas große Unterschiede in der generellen Offenheit gegenüber Migration und bei der Frage, welche Kriterien Migrantinnen und Migranten für vollständige Inklusion erfüllen

\footnotetext{
3 Bei Polen und Ungarn wird häufig auch von mittelosteuropäischen Ländern gesprochen. Wir richten uns nach der statistischen Länderklassifikation der Vereinten Nationen (2020), bei der diese beiden Länder zu Osteuropa zählen.
} 
sollten. Am liberalsten sind nord- und westeuropäische Länder, während sich osteuropäische Länder als deutlich migrationsskeptischer erweisen (Heath und Richards 2020). In Ländern mit einem niedrigeren ökonomischen Niveau und einem höheren Niveau autoritärer Einstellungen - wie den osteuropäischen Ländern - sind nicht nur die Vorbehalte am größten, sondern auch die über Bildung gemessenen Einstellungsunterschiede am kleinsten (Eick 2020). Insgesamt scheinen der sozioökonomische Cleavage und die Polarisierung bei der Migrationsfrage in migrationsfreundlichen Ländern größer zu sein als in restriktiven Ländern (Heath und Richards 2020).

Auch für die Wir-Sie-Ungleichheiten lässt sich ein sozialer Gradient ausmachen. So sind Angehörige der oberen Berufsklassen toleranter gegenüber Homosexuellen (Andersen und Fetner 2008). Wie auch bei der Migrationsfrage ist der Zusammenhang zwischen Bildung und Toleranz in insgesamt toleranteren Ländern stärker ausgeprägt; die Statusgruppendifferenzen sind also größer (Dotti Sani und Quaranta 2020). Im Ländervergleich sind Fragen der Modernisierung, der Religion und der kulturellen Tradition als wichtige Determinanten ausgemacht worden (Gerhards 2010; van den Akker et al. 2013). Osteuropäische Länder weisen hierbei einen geringeren Grad an Toleranz gegenüber Homosexualität auf als westeuropäische Länder (Fitzgerald et al. 2014; Röder und Lubbers 2015).

Die wenigsten empirischen Vorarbeiten finden sich $\mathrm{zu}$ den Heute-Morgen-Ungleichheiten. Erste Analysen deuten darauf hin, dass die Themen Ökologie und Nachhaltigkeit besonders stark von den oberen Einkommens- und Bildungsgruppen getragen werden (Otto und Gugushvili 2020; Fritz und Koch 2019). Zugleich ist aber die Annahme problematisch, dass die unteren Schichten kein Interesse an dem Thema hätten (Neckel 2020). Die nordischen Länder erweisen sich als besonders klimafreundlich. Hier sind ressourcenschonende Verhaltensweisen weit verbreitet, und relativ viele Menschen machen sich Sorgen um den Klimawandel (Europäische Kommission 2008). In postsozialistischen Ländern finden sich solche Sorgen vergleichsweise seltener (Franzen und Vogl 2013). Auch die Bereitschaft, sich gegen den Klimawandel zu engagieren, ist hier eher schwach ausgeprägt (Chaisty und Whitefield 2015). In Bezug auf das Verhältnis von sozialen und ökologischen Fragen sind die Ergebnisse nicht vollständig konklusiv. In einigen Ländern sind pro-soziale und pro-ökologische Orientierungen positiv verknüpft, in anderen nicht; einige Studien identifizieren gruppenspezifische Einstellungsmixe (Otto und Gugushvili 2020; Fritz und Koch 2019; Jakobsson et al. 2018). Zur Frage, inwieweit sich der soziale Gradient ökologischer Einstellungen zwischen Ländern unterscheidet, gibt es bislang wenig Forschung. Tranter und Booth (2015) können allerdings zeigen, dass die Bildungsunterschiede in der Sorge um den Klimawandel relativ unsystematisch zwischen den Ländern variieren.

Der Literaturreview zeigt, dass sich im Ländervergleich nicht nur Niveauunterschiede in der Befürwortung oder Ablehnung der Themen ergeben, sondern auch, dass der soziale Gradient variiert. Bei den Wir-Sie-Ungleichheiten und den InnenAußen-Ungleichheiten sollten sich insbesondere Länder aus Osteuropa durch defensivere Haltungen von den übrigen Ländern abheben, auch die Gruppendifferenzen sollten hier geringer sein. Für die Oben-Unten-Ungleichheiten sind starke Präferenzen für Umverteilung insbesondere in osteuropäischen Ländern zu erwarten, die zusammen mit den skandinavischen Ländern auch die deutlichsten Statusgruppen- 
differenzen aufweisen dürften. Bei den Heute-Morgen-Ungleichheiten sollten insbesondere in Skandinavien pro-ökologische Haltungen weit verbreitet sein.

\section{Länderauswahl und Forschungsfragen}

Für unsere Analysen haben wir sechs EU-Länder ausgewählt, die sich im Hinblick auf die hier untersuchten Themenkomplexe politisch und institutionell unterscheiden und ein breites Spektrum abdecken (für eine Übersicht siehe Tabelle 1 und die dort angegebene Literatur): Mit Ungarn und Polen beziehen wir zwei postsozialistische Länder ein, in denen die Einkommensungleichheit - ausgehend von einem sehr niedrigen Niveau direkt nach dem Fall des Eisernen Vorhangs - auf einem mittleren Niveau liegt und die eher defensiv bis ablehnend bei der Integration von Migranten agieren. LGBT-Personen (lesbian, gay, bisexual, transgender) werden wenig bis kaum akzeptiert. In beiden Ländern ging der jüngste Abbau von Minderheitenrechten mit einer umfassenden anti-liberalen Politik der rechtskonservativen bzw. rechtspopulistischen Regierungen einher (Einschränkung von Pressefreiheit und Rechtsstaatlichkeit). Der Klimaschutz wird politisch vergleichsweise wenig priorisiert. Auch Italien zeichnet sich durch eine eingeschränkte Akzeptanz von LGBT-Personen aus. Die Migrationspolitik und der Klimaschutz sind moderat ausgeprägt, allerdings ist die Einkommensungleichheit recht hoch und rangiert innerhalb der EU im oberen Mittelfeld.

Schweden steht in vielen Hinsichten in einem starken Kontrast zu diesen Ländern. Geprägt durch einen stark umverteilenden Wohlfahrtsstaat ist die Einkommensungleichheit vergleichsweise gering, und es existiert institutionell eine relativ große Offenheit für die Aufnahme und Integration von Zuwanderern. Bei Fragen der Akzeptanz von LGBT-Personen gilt Schweden als ein historischer Vorreiter der Liberalisierung, zudem zählt die Klimapolitik des Landes zu den umweltfreundlichsten der Welt.

Tab. 1 Länderübersicht (Quelle: Eigene Darstellung)

\begin{tabular}{lllllll}
\hline & HU & PL & IT & FR & DE & SE \\
\hline $\begin{array}{l}\text { Einkommensungleichheit } \\
\text { (Gini nach Umverteilung 2015) }\end{array}$ & 0,284 & 0,291 & 0,333 & 0,295 & 0,293 & 0,278 \\
$\begin{array}{l}\text { LGBT-Akzeptanz-Index } \\
\text { (2009-2013) }\end{array}$ & 3,90 & 3,99 & 4,77 & 5,74 & 5,62 & 6,55 \\
$\begin{array}{l}\text { „Migrant Integration Policy“-Index } \\
\text { (2015) }\end{array}$ & 45 & 41 & 59 & 54 & 61 & 78 \\
Klimaschutz-Index (2018) & 44 & 47 & 60 & 60 & 57 & 74 \\
\hline
\end{tabular}

Einkommensungleichheit berichtet Daten nach OECD (2020).

Der LGBT-Akzeptanz-Index basiert auf gemittelten Einstellungsdaten aus 11 unterschiedlichen Bevölkerungsumfragen (Flores und Park 2018).

Der „Migrant Integration Policy“-Index misst die Politik zur Integration von Migrantinnen und Migranten in den Bereichen Aufenthaltsmöglichkeiten, Familienzusammenführung, Einbürgerungsmöglichkeiten, Antidiskriminierung, Arbeitsmarktpartizipation, Bildung, Gesundheit und politische Partizipationsmöglichkeiten; Daten aus dem Jahr 2014 (MIPEX 2015).

Der Klimaschutz-Index bildet die Dimensionen Klimapolitik, Treibhausgasemissionen, Energieverbrauch und Anteil erneuerbarer Energien ab (Burck et al. 2017). 
Mit Deutschland und Frankreich werden zudem die beiden bevölkerungsreichsten Länder der EU aufgenommen. Beide Länder bewegen sich auf einem mittleren Niveau der Einkommensungleichheit, und auch der Klimaschutz, die Offenheit für Migration und die Akzeptanz von LGBT-Personen erreichen in beiden Ländern ein mittleres Niveau. Allerdings ist Deutschland bei Fragen der Migration etwas progressiver als Frankreich, das wiederum etwas mehr Offenheit für die Akzeptanz von LGBT-Personen und einen leichten Vorsprung beim Klimaschutz aufweist.

Mit der oben ausbuchstabierten konzeptionellen Ausrichtung und dem heterogenen Ländersample wollen wir nun folgende Fragen, welche die Zwei-WeltenTheorie als Bezugspunkt haben, beantworten:

1. Welche Dimensionierungen und Zusammenhänge finden sich zwischen den Einstellungsbereichen?

2. Wo sind die Länder bezüglich dieser Einstellungen positioniert, und wie sieht die Verteilung dieser Einstellungen in der jeweiligen Bevölkerung aus? Wie hängen Einstellungen und Ungleichheitspositionen zusammen?

3. Wie stark ist die vertikale Strukturierung der Einstellungen im Vergleich zu anderen, horizontalen Strukturdeterminanten?

Was den Fallvergleich angeht, versuchen wir uns nicht an einer kausalen Fragestellung, sondern verbleiben auf der deskriptiv-explorativen Ebene. Die Analysen sind nicht hypothesentestend, sondern können allenfalls zur Ableitung von Hypothesen genutzt werden. Übergeordnete Perspektive unserer Datenanalyse ist die Frage danach, ob wir tatsächlich von konsistenten Einstellungssyndromen ausgehen können und ob wir über die Einstellungen hinweg - in plakativer Zuspitzung formuliert eine Lagerbildung in ein „kosmopolitisches Oben“ und ein „kommunitaristisches Unten“ beobachten können.

\section{Daten und Methoden}

Für unsere Analysen nutzen wir die Daten der achten Welle des European Social Survey (ESS), die für unser Ländersample zwischen August 2016 und November 2017 erhoben wurden. Beim ESS handelt es sich um eine alle zwei Jahre stattfindende Erhebung, bei der in zahlreichen europäischen Ländern die in Privathaushalten lebende Bevölkerung ab einem Alter von 15 Jahren in persönlichen Face-to-Face-Interviews zu ihren Einstellungen und sozialstrukturellen Hintergründen befragt wird (für nähere Informationen zu dieser Erhebung siehe die Dokumentation European Social Survey 2018). In der achten Welle wurden in den wechselnden Frageschwerpunkten dieser Erhebung alle von uns anvisierten Einstellungskomplexe einbezogen, sodass sich diese Daten für unsere Untersuchung besonders gut eignen.

Konkret nutzen wir für die Einstellungsmessung unserer Ungleichheitsachsen die Zustimmung der Befragungspersonen zu folgenden Aussagen bzw. ihre Antwort auf folgende Fragen:

1. Der Staat sollte Maßnahmen ergreifen, um Einkommensunterschiede zu verringern. 
2. Damit eine Gesellschaft gerecht ist, sollten die Unterschiede im Lebensstandard der Menschen gering sein.

3. Schwule und Lesben sollten ihr Leben so führen dürfen, wie sie es wollen.

4. Schwule und lesbische Paare sollten die gleichen Rechte haben, Kinder zu adoptieren, wie Paare, die aus Mann und Frau bestehen.

5. Was würden Sie sagen, ist es im Allgemeinen gut oder schlecht für die deutsche ${ }^{4}$ Wirtschaft, dass Zuwanderer hierher kommen?

6. Würden Sie sagen, dass das kulturelle Leben in Deutschland im Allgemeinen durch Zuwanderer untergraben oder bereichert wird?

7. Wird Deutschland durch Zuwanderer zu einem schlechteren oder besseren Ort zum Leben?

8. Wie sehr fühlen Sie sich persönlich verantwortlich, einen Beitrag zu leisten, damit der Klimawandel reduziert wird?

9. Wie besorgt sind Sie über den Klimawandel?

Aus konzeptioneller Sicht sollten sich die ersten beiden Items auf Oben-UntenUngleichheiten beziehen, die nächsten beiden Items sollten Wir-Sie-Ungleichheiten messen und die Fragen fünf bis sieben Innen-Außen-Ungleichheiten. ${ }^{5}$ Aspekte der Heute-Morgen-Ungleichheiten sollten über die letzten beiden Fragen abgebildet werden. Die Aussagen bzw. Fragen konnten auf unterschiedlich langen Skalen beantwortet werden. Für die ersten vier Aussagen stand eine 5-stufige Antwortskala zur Verfügung (,stimme stark zu“, ,stimme zu“, ,,weder noch“, ,lehne ab“, „lehne stark $\left.a b^{\prime \prime}\right)$. Die nachfolgenden vier Fragen hatten eine 11-stufige Antwortskala, bei der jeweils nur die Skalenenden benannt wurden (,schlecht für die Wirtschaft" und ,gut für die Wirtschaft“; ,kulturelles Leben wird untergraben“ und „kulturelles Leben wird bereichert“; ,wird zu einem schlechteren Ort“" und ,wird zu einem besseren Ort“; ,überhaupt nicht“ und ,sehr stark“). Bei der letzten Frage wurde eine Antwortskala mit fünf Ausprägungen genutzt (,,überhaupt nicht besorgt“, ,,nicht sehr besorgt", ,,etwas besorgt", ,,sehr besorgt", ,,äußerst besorgt"). Für eine bessere Vergleichbarkeit und Übersichtlichkeit haben wir die Antwortskalen so transformiert, dass sie einheitlich von -2 bis +2 reichen (eine detaillierte Übersicht über die Rekodierungen findet sich in Tabelle 4 im Anhang). ${ }^{6}$ Hierbei stehen hohe positive Werte für eine starke Kritik an ökonomischer Ungleichheit, eine hohe Anerkennungsbereitschaft gegenüber sexuellen Minderheiten, integrationsfreundliche Haltungen und

\footnotetext{
${ }^{4}$ In der ESS-Erhebung waren die Fragen mit Länderbezug (Fragen 5-7) auf das jeweilige Erhebungsland bezogen.

5 In unserer vorangegangenen Studie zum Thema konnten wir die Inklusion von Migranten in die Sozialleistungssysteme als Item mit aufnehmen, was hier nicht der Fall ist (Mau et al. 2020).

6 Wir orientieren uns hierbei an der Vorgehensweise von Proto und Rustichini (2012). Konkret transformieren wir die 11 er-Antwortskalen mit der Formel $(X-5) * 4 / 10$, und wir transformieren die 5er-Antwortskalen über die Formel X-3. Die Antwortskala der Items 1, 2, 3 und 4 wurde zuvor invertiert. Die beiden Fragen zum Klimawandel wurden über eine Filterführung nur jenen Personen gestellt, die glauben, der Klimawandel existiere. Jene, die dies nicht glauben, werden von uns der Kategorie ,fühle mich überhaupt nicht verantwortlich“ bzw. ,bin überhaupt nicht besorgt“ zugeordnet, da wir davon ausgehen, dass diese Personen - aufgrund ihrer Negierung des Klimawandels - hier weder eine persönliche Verantwortung sehen noch einen Grund zur Sorge.
} 
ein ausgeprägtes Umweltbewusstsein. Negative Werte bringen hingegen eine Ungleichheitstoleranz in der jeweiligen Dimension zum Ausdruck. Ein Wert von Null steht für indifferente Haltungen. ${ }^{7}$

Zur Messung der Statuslage verwenden wir Informationen zur Bildung, zur Klassenzugehörigkeit und zur subjektiven Beurteilung des Haushaltseinkommens. Die Bildung bezieht sich auf die International Standard Classification of Education (ISCED) und weist vier Kategorien auf: gering (ISCED I/II), mittel (ISCED III), mittel-gehoben (ISCED IV) und hoch (ISCED V). Die Klassenzugehörigkeit wird über das Klassenschema von Daniel Oesch dargestellt, wobei wir die 5er-Kategorisierung dieses Schemas verwenden und zwischen oberer Dienstklasse, unterer Dienstklasse, kleinen Selbstständigen, Facharbeitern und ungelernten Arbeitern unterscheiden (Oesch 2006). Für die Zuordnung maßgeblich ist der aktuelle Beruf der Befragungsperson bzw. (bei fehlenden Informationen zum eigenen Beruf) der aktuelle Beruf des Partners/der Partnerin. Für die subjektive Beurteilung des Haushaltseinkommens verwenden wir die Einschätzung der Befragungsperson, ob sie bzw. der Haushalt mit dem Einkommen bequem leben kann oder zurechtkommt oder nur (sehr) schwer zurechtkommt. Für die Analyse der horizontalen Strukturgeber von Einstellungen verwenden wir das Geschlecht (Mann, Frau), das Alter (15-24, 25-39, 40-64, 65 oder älter) sowie den selbst eingeschätzten Urbanitätsgrad des Wohnortes (Großstadt, Vorort/Randgebiet einer Großstadt, Stadt/Kleinstadt, Dorf/Bauernhof/Haus auf dem Land). Ein Überblick über die Rekodierungen dieser Variablen sowie die Besetzung der Kategorien in den jeweiligen Ländern findet sich in Tabelle 5 im Anhang. In die Untersuchung nehmen wir alle Fälle auf, die in den abhängigen und unabhängigen Variablen keine fehlenden Werte aufweisen. ${ }^{8}$ Auf diese Weise stehen uns für die sechs ausgewählten Länder 10.580 Untersuchungspersonen zur Verfügung, wobei die länderspezifische Fallzahl von 1178 (in Ungarn) bis 2698 (in Deutschland) reicht.

Neben einfachen deskriptiven Analysen kommen im Folgenden explorative Faktorenanalysen und Regressionsanalysen zur Anwendung. Genauere Hinweise zu diesen Verfahren und zur Interpretation der zugehörigen Ergebnisse geben wir bei der Präsentation der jeweiligen Befunde. Alle Analysen wurden, sofern nicht explizit anders ausgewiesen, mit den vom Team des ESS bereitgestellten Anpassungsgewichten (post-stratification weight including design weight) gewichtet (European Social Survey 2018; Kaminska 2020). Für die länderübergreifende Faktorenanalyse wurden diese Gewichte so reskaliert, dass jedes Land unabhängig von seiner Größe den gleichen Einfluss hat.

\footnotetext{
7 Hiervon wird nur in einem Fall abgewichen: Bei der Frage ,Wie besorgt sind Sie über den Klimawandel?" steht der Wert 0 für die Antwort ,etwas besorgt“, weil bei der zugehörigen Antwortskala keine Kategorie für Indifferenz vorhanden ist. Da es sich bei dieser Antwort allerdings um die mittlere Kategorie auf einer 5-stufigen Antwortskala handelt und sie die empirische Modalkategorie der Antworten darstellt, ist es nicht unplausibel anzunehmen, dass indifferente Befragungspersonen diese Kategorie gewählt haben.

8 Hiervon weichen wir nur in einem Fall ab: Aufgrund von relativ vielen fehlenden Werten zur Berufsklasse weisen wir Missings in dieser Variable als separate Kategorie aus und nehmen die entsprechenden Fälle dadurch mit in die Analysen auf.
} 


\section{Ergebnisse}

In einem ersten Schritt geht es um die Frage, inwieweit sich Kommunitaristen und Kosmopoliten nicht nur idealtypisch charakterisieren lassen, sondern auch empirisch anhand von Einstellungen beobachtbar sind. Wenn sich die These, dass Gegenwartsgesellschaften von einer entsprechenden Spaltungslinie durchzogen seien, nicht lediglich auf diskursive Phänomene, die Parteienlandschaft oder wortstarke Mikromilieus bezieht, dann sollten sich auch - zumindest in einer gewissen Ausprägung entsprechend konturierte Profile bei den Einstellungen zu den von uns untersuchten Themenbereichen finden: Jene, die ökonomische Umverteilung stark stützen, aber sehr zurückhaltend beim Klimaschutz, bei der Aufnahme von Migranten und bei der Anerkennung von Homosexuellen sind. Und jene, deren Einstellungsprofil entgegengesetzt strukturiert ist, die also ein ausgeprägtes Umweltbewusstsein, eine positive Sicht auf Migranten und auf die Anerkennung sexueller Diversität haben, bei gleichzeitig geringer Priorisierung ökonomischer Umverteilung.

Definiert man die Zugehörigkeit zu den Gruppen der Kommunitaristen und Kosmopoliten über solche klar divergierende, letztlich stark ausdefinierte Einstellungsprofile, lassen sich in den ausgewählten Ländern allenfalls zwischen 5 Prozent (Schweden, Frankreich und Deutschland) und 17 Prozent (Ungarn) der Bevölkerung diesen beiden Gruppen zuordnen. ${ }^{9}$ Der weitaus größte Teil der Bevölkerung (zwischen 95 und 83 Prozent) würde in einer solchen - zugegebenermaßen zugespitzten Klassifikation als Mischtyp gelten, bei dem die Einstellungen weniger klar konturiert sind als oft in der Diskussion behauptet (siehe linker Teil von Abbildung 1). Bei diesem Mischtyp würden dann beispielsweise umverteilungsaffine Haltungen mit einem Mix aus befürwortenden und ablehnenden Positionen zu den neueren Ungleichheitsthemen einhergehen. Doch selbst wenn man davon ausgeht, dass sich der Konflikt zwischen Kommunitaristen und Kosmopoliten nur auf die neueren Ungleichheitsfragen bezieht, und man demzufolge die beiden Items zu ökonomischer Ungleichheit aus der Gruppendefinition ausschließt, bleibt dieser prinzipielle Befund im Wesentlichen stabil (siehe rechter Teil von Abbildung 1). Dann lassen sich zwar mehr Personen den Gruppen der Kommunitaristen und der Kosmopoliten zuordnen, wobei insbesondere der deutlich größere Anteil an Kosmopoliten in Westund Nordeuropa ins Auge sticht. Aber selbst unter einer solchen weniger restriktiven Klassifikation würde in jedem der untersuchten Länder die Mehrheit der Bevölkerung zum Mischtyp zählen (zwischen 54 Prozent in Schweden und 79 Prozent in Polen). Es scheint vor dem Hintergrund dieser Daten so zu sein, dass die Pole recht schwach besetzt sind und die Realtypen mit den (idealtypischen) Beschreibungen erst einmal wenig zu tun haben. Eine Zwei-Lager-Welt lässt sich in diesen Daten erst einmal nicht finden.

Bereits eine solch einfache Deskription zeigt also, dass die Einstellungsprofilierung der Bevölkerung komplexer ist, als es die These der Bifurkation oder Spaltung

\footnotetext{
9 Als Kommunitaristen zählen für uns in diesen Analysen jene Personen, die im Mittel der Items zu ökonomischer Umverteilung ungleichheitskritische Haltungen äußern und jeweils im Mittel der Items zu Migration, zum Klimawandel und zur Anerkennung Homosexueller ablehnende Positionen vertreten. Kosmopoliten sind für uns jene mit einem umgekehrten Einstellungsmuster.
} 


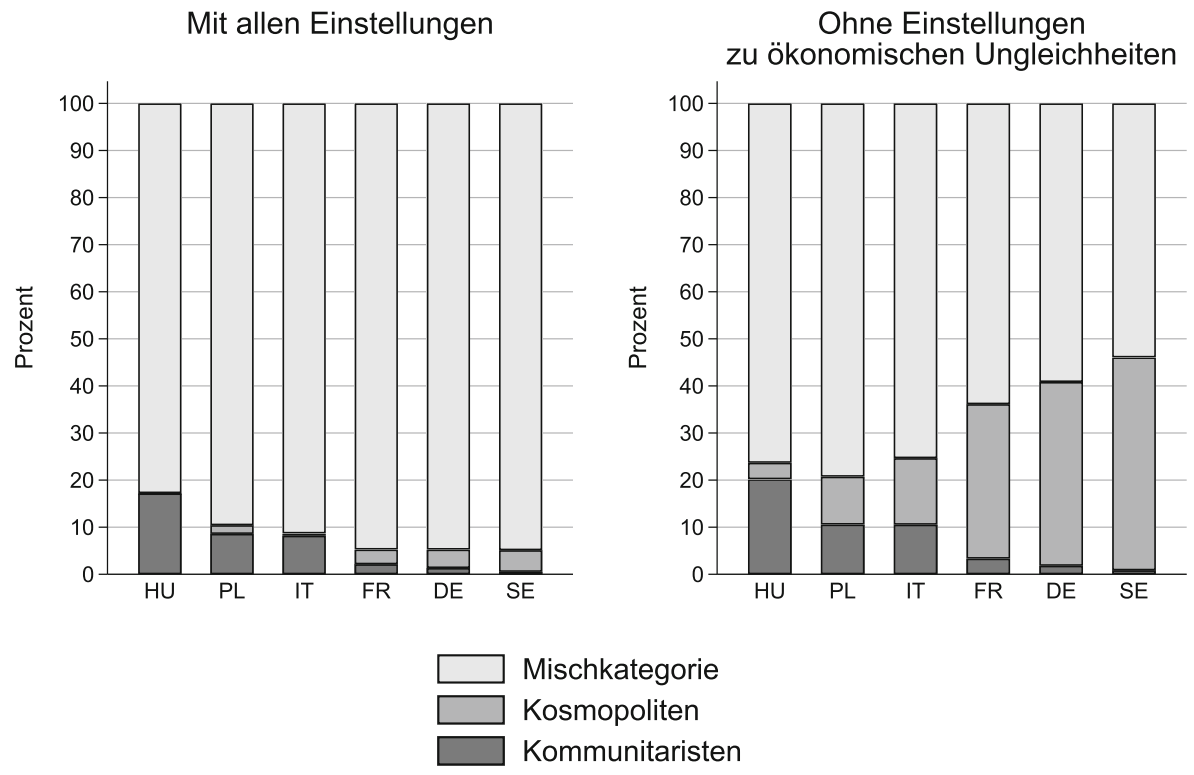

Abb. 1 Gruppengrößen bei Definition der Gruppen über klar divergierende Einstellungsprofile (Datenbasis: ESS 2016; eigene Berechnungen, gewichtet. Für die Gruppendefinition siehe Fußnote 9.)

zwischen Kommunitaristen und Kosmopoliten nahelegt. Deshalb wollen wir nun etwas genauer unter die Lupe nehmen, wie sich die Einstellungsthemen zueinander verhalten, ob und - wenn ja - bei welchen Themen die Einstellungen tatsächlich polarisiert sind und wo sich soziale Statusgruppen innerhalb der jeweiligen Themen positionieren. Wir kommen nun also zur Beantwortung der oben aufgestellten Forschungsfragen.

Im ersten Schritt werden wir ermitteln, wie sich die Einstellungsthemen zueinander verhalten, oder genauer: welche Einstellungsdimensionierung sich in den untersuchten Ländern empirisch auffinden lässt. Zeigt sich tatsächlich eine zweidimensionale Welt der Einstellungen, bei denen sich Positionen zu ökonomischen Themen klar von solchen zu soziokulturellen Themen abgrenzen lassen (wobei Letztere so unterschiedliche Aspekte wie Migration, sexuelle Diversität und Klimaschutz umfassen, die dann tatsächlich als ein übergreifender Einstellungskomplex aufzufassen wären)? Zur Beantwortung dieser Frage verwenden wir explorative Faktorenanalysen. Dieses statistische Verfahren erlaubt zwar keine definitive Antwort im Sinne eines Hypothesentests, aber es bietet - unter Verwendung gängiger Schwellenwerte - einen Überblick der Zusammenhänge und ermöglicht so eine erste Beurteilung. Konkret kann mit diesem Verfahren ermittelt werden, welche und wie viele - nur schwer direkt messbare - Einstellungen (z. B. „links“ und „rechts“, „kosmopolitisch“ und „kommunitaristisch“) einem Set an Antworten auf themenverwandte 
Fragen unterliegen (Bartholomew et al. 2008, S. 175). Die Faktorenanalyse ${ }^{10}$ führen wir separat für die einzelnen Länder und gepoolt über alle Länder hinweg durch, wobei jedes Land einen gleich großen Einfluss auf das Ergebnis hat.

Die Parallelanalyse (Horn 1965) ist ein statistisches Mittel, um im Rahmen einer Faktorenanalyse festzustellen, wie viele den Items unterliegende Dimensionen die Datenstruktur am besten beschreiben. Sie zeigt in unserem Fall an, dass im gepoolten Sample eine Lösung mit vier Faktoren optimal ist. Die neun Einstellungsvariablen lassen sich demnach am besten beschreiben, wenn man von vier darunterliegenden Einstellungskomplexen (Faktoren) ausgeht. ${ }^{11}$

Die themenverwandten Items laden dabei jeweils auf einen Faktor, was insgesamt die von uns angedachte konzeptionelle Zuordnung abbildet und unserem Vorgängerartikel entspricht, der mit anderen Daten arbeitet (siehe Tabelle 2; Mau et al. 2020). Querladungen bestehen zwischen den Einstellungen zur Migration und dem Wir-Sie-Faktor, sie bleiben aber unter dem in der Literatur üblichen Schwellenwert von 0,3 . Hier könnte also durchaus zumindest zum Teil von einem Zusammenhang ausgegangen werden. Unsere Analysen sprechen jedoch dafür, dass hier eher die Unterschiede als die Gemeinsamkeiten der Dimensionen zu betonen sind. Auch unsere länderspezifischen Faktorenanalysen kommen zu relativ eindeutigen 4-Faktoren-Lösungen (siehe Tabelle 6 im Anhang). Lediglich in Italien lädt das Item zum Adoptionsrecht mit 0,33 auch auf dem Innen-Außen-Faktor. Wir finden insgesamt also keine zweidimensionale Welt der Einstellungen, sondern vier relativ eigenständige Einstellungsdimensionen, die der von uns vorgeschlagenen Konzeption der vier Ungleichheitsachsen entsprechen. Aufgrund dieser relativ klaren empirischen Dimensionierung der Einstellungen erstellen wir für die weiteren Analysen vier Summenindizes. Bei diesen werden die Items der jeweiligen Dimensionen addiert und durch die Anzahl der addierten Items dividiert. Die so erstellten Indizes besitzen die gleiche Skalenlänge wie die (von uns transformierten) Ausgangsitems ( -2 bis +2 ), wobei erneut hohe positive Werte eine starke Ungleichheitskritik, eine hohe Anerkennungsbereitschaft gegenüber sexuellen Minderheiten, positive Haltungen zur Migration und ein ausgeprägtes Umweltbewusstsein anzeigen. ${ }^{12}$

Wo liegen nun die Länder auf diesen Einstellungsdimensionen? Bei Fragen der ökonomischen Oben-Unten-Ungleichheiten sowie bei den ökologischen Heute-Mor-

\footnotetext{
${ }^{10}$ Wir verwenden jeweils eine orthogonale Varimax-Rotation. Eine schiefwinkelige Rotation (ObliminVerfahren) führt zu den gleichen Kernaussagen. Auch die Hauptachsenmethode und Weighted-Least-Squares-Methode führen zu den gleichen Kernaussagen. Die Ergebnisse dieser hier nicht gezeigten Analysen sind auf Anfrage bei den Autoren erhältlich.

${ }^{11}$ Es muss betont werden, dass dieses Ergebnis (wie bei allen Faktorenanalysen) von der Formulierung und der theoretisch begründeten Vorauswahl der Items beeinflusst ist. Es sagt nicht, dass es nur oder genau vier Einstellungsdimensionen gibt. Bei der Betrachtung nur einer Dimension, mit deutlich mehr Items, die verschiedene Facetten abdecken, würde man möglicherweise mehr Diversität mit wiederum feineren unterliegenden (Unter-)Dimensionen entdecken. Die hier gezeigte breitere Analyse liefert jedoch das wichtige Ergebnis, dass die „,neuen“ Konflikte nicht alle durch die gleiche latente Eigenschaft (,,anywheres/ somewheres“, „Kosmopolitismus/Kommunitarismus“) zu erklären sind.

12 Die Maße der Skalenkonsistenz (Cronbachs Alpha) für die Indizes sind: Oben-Unten $(0,54)$, Wir-Sie $(0,69)$, Innen-Außen $(0,88)$ und Heute-Morgen $(0,62)$. Obgleich dieses Maß insbesondere für den Index der Oben-Unten-Ungleichheiten - auch aufgrund der geringen Zahl der einbezogenen Items - recht gering ist, wird in keiner Dimension ein Wert unterschritten, der eine Indexbildung ausschließen würde.
} 
Tab. 2 Ladung der Items auf den Faktoren. Faktorenanalyse mit gepooltem Sample, orthogonale Varimax-Rotation (Datenbasis: ESS 2016; eigene Berechnungen, gewichtet)

\begin{tabular}{lcccc}
\hline & $\begin{array}{l}\text { Oben-Unten- } \\
\text { Ungleichheiten }\end{array}$ & $\begin{array}{l}\text { Innen-Außen- } \\
\text { Ungleichheiten }\end{array}$ & $\begin{array}{l}\text { Wir-Sie-Un- } \\
\text { gleichheiten }\end{array}$ & $\begin{array}{l}\text { Heute-Morgen- } \\
\text { Ungleichheiten }\end{array}$ \\
\hline $\begin{array}{l}\text { Einkommensunterschiede } \\
\text { sollten gering sein }\end{array}$ & $\mathbf{0 , 6 0}$ & 0,02 & 0,00 & 0,03 \\
$\begin{array}{l}\text { Umverteilung durch Staat } \\
\text { Toleranz gegenüber }\end{array}$ & $\mathbf{0 , 6 2}$ & $-0,07$ & $-0,04$ & 0,01 \\
$\begin{array}{l}\text { Homosexuellen } \\
\text { Adoptionsrecht gleichge- }\end{array}$ & $-0,07$ & 0,22 & $\mathbf{0 , 7 1}$ & 0,10 \\
schlechtlicher Paare & & 0,23 & $\mathbf{0 , 7 0}$ & 0,11 \\
$\begin{array}{l}\text { Migration, wirtschaftlich } \\
\text { Migration, kulturell }\end{array}$ & $-0,05$ & $\mathbf{0 , 7 9}$ & 0,19 & 0,11 \\
$\begin{array}{l}\text { Migration, allgemein } \\
\text { Persönliche Verantwor- }\end{array}$ & $-0,03$ & $\mathbf{0 , 8 2}$ & 0,23 & 0,09 \\
tung Klimawandel & $-0,04$ & $\mathbf{0 , 8 4}$ & 0,18 & 0,06 \\
Sorgen Klimawandel & 0,08 & 0,20 & 0,13 & $\mathbf{0 , 6 7}$ \\
\hline
\end{tabular}

Fett formatiert dargestellt sind Faktorladungen $\geq 0,3$.

gen-Ungleichheiten finden wir nur sehr begrenzte Länderunterschiede (siehe Abbildung 2). Zwar schaut die Bevölkerung in Ungarn und Italien etwas kritischer auf Ungleichheiten in Einkommen und Lebensstandard, und Befragte in Frankreich und Deutschland sind beim Klimawandel im Mittel ein wenig besorgter und verantwortungsbewusster als die Bevölkerung in den anderen Ländern, aber diese Unterschiede sind nicht besonders stark ausgeprägt. Deutliche Länderunterschiede ergeben sich allerdings im Bereich der Innen-Außen-Ungleichheiten, bei dem sich Schweden sowie - etwas abgeschwächter - Deutschland und Polen als eher migrationsoffen zeigen, während Ungarn und Italien migrationskritischer positioniert sind. Auffällig ist, dass die Einstellungen zur Migration sehr stark über die ablehnenden und bejahenden Bereiche der Antwortskala streuen - ein möglicher Hinweis auf Polarisierungen. ${ }^{13}$ Am deutlichsten sind die Länderunterschiede im Bereich der WirSie-Ungleichheiten, bei denen die Befragten in Schweden, Deutschland und Frankreich mehrheitlich der Meinung sind, dass sexuelle Minderheiten anerkannt werden sollten, während in Ungarn und Polen die Haltung dominiert, diesen Minderheiten die Anerkennung zu verwehren. Auch für diese Dimension können wir teilweise recht ausgeprägte Streuungen beobachten.

Betrachtet man die übergreifende Einstellungsprofilierung der Länder, dann haben wir auf der einen Seite Deutschland und Schweden, wo die Bevölkerung in allen Einstellungsdimensionen im Mittel eher ungleichheitskritisch positioniert ist. Dies gilt mit Ausnahme der eher unentschiedenen Haltung bei Migrationsfragen auch für Frankreich. Die Bevölkerung Ungarns unterscheidet sich mit ihren ablehnenden

\footnotetext{
13 Es sei hier darauf hingewiesen, dass insbesondere die Items zur Migration mit einer längeren Antwortskala versehen waren (11er-Skala). Diese Skala wurde von uns zwar auf den Wertebereich -2 bis 2 transformiert, dennoch weist die so modifizierte Antwortskala mehr Zwischenabstufungen auf, als die von uns ebenfalls verwendeten 5er-Skalen. Wir gehen davon aus, dass dies im vorliegenden Fall nicht zu methodischen Artefakten führt, da unsere Befunde sehr stark jenen ähneln, die von uns in Deutschland auf der Basis von Items mit gleicher Skalenlänge erzielt wurden (Mau et al. 2020).
} 

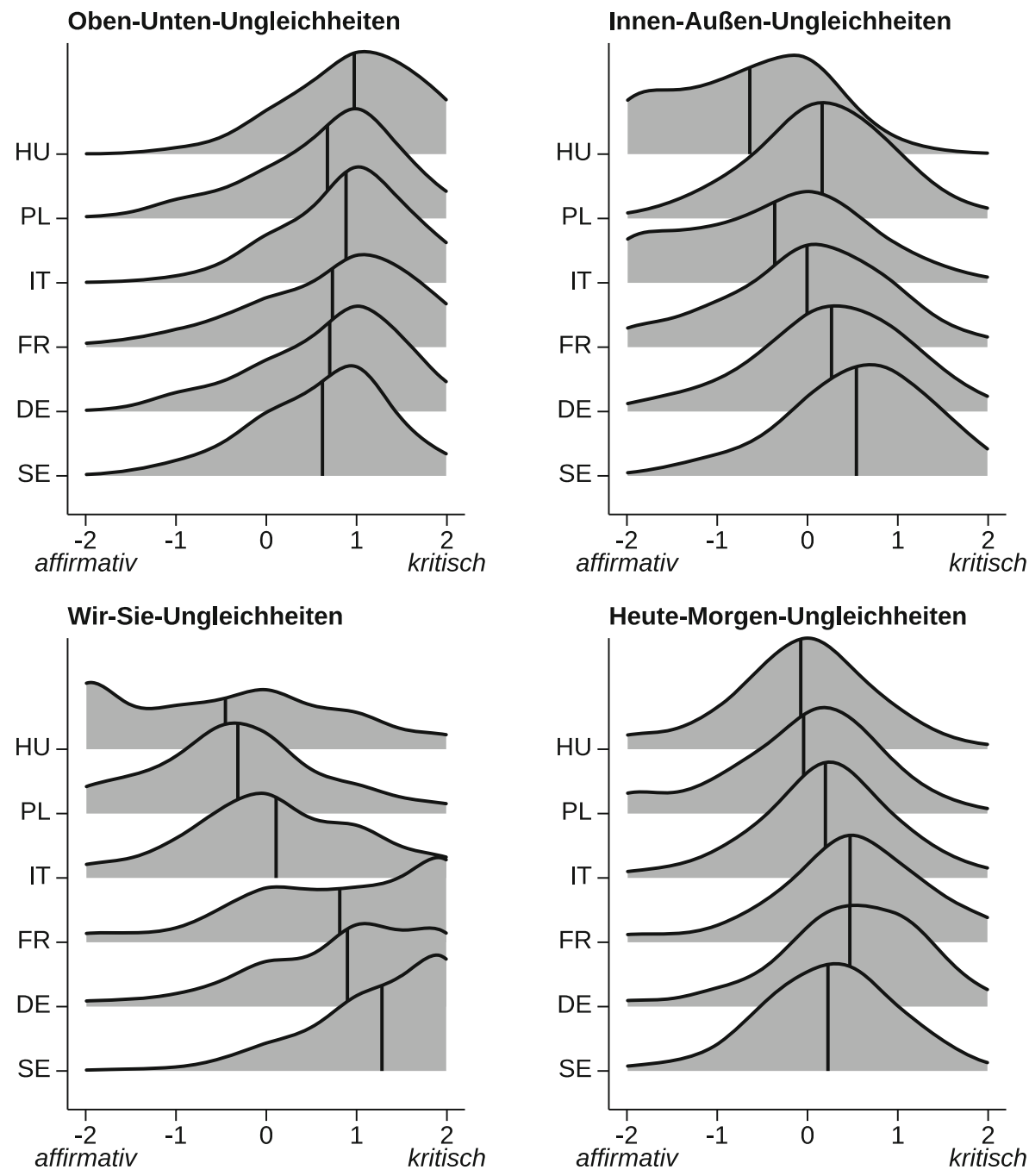

Abb. 2 Mittelwerte und Verteilungen der Einstellungsindizes (Datenbasis: ESS 2016; eigene Berechnungen, gewichtet)

Haltungen bei den neueren Ungleichheitsthemen recht stark von diesen Ländern. Polen und Italien liegen einstellungsmäßig zwischen diesen Gruppen. Auffällig sind hier aber insbesondere die migrationskritischen Haltungen in Italien und die relativ stark ablehnenden Positionen zu sexueller Diversität in Polen.

Insgesamt kann festgehalten werden, dass sich eine deutliche Ablehnung von staatlicher Umverteilung in keinem unserer Fallländer findet, ebenso wenig Extrempositionen bei den ökologischen Einstellungen, wo sich jeweils mittige Antwortverteilungen ergeben. Bei der Migrationsfrage und der Anerkennungsthematik gibt es hingegen starke länderspezifische Unterschiede. In Schweden finden wir eine starke Tendenz in Richtung der sozialen und rechtlichen Anerkennung sexueller Diversi- 
tät, auch in Deutschland und Frankreich ist der anerkennungsbereite Pol recht stark besetzt, während Ungarn und Polen diesbezüglich stark polarisiert sind. Dort finden sich auch relativ große Gruppen auf dem Extrempol der Ablehnung. Hervorzuheben sind auch die Einstellungen zu Innen-Außen-Ungleichheiten in Italien: Die Verteilung ähnelt am liberalen Ende der in Frankreich, es gibt aber eine sehr viel größere Zahl extrem migrationskritischer „Demarkationisten“.

Nachdem wir gezeigt haben, dass sich die von uns ausgewählten Länder hinsichtlich der Einstellung ihrer Bevölkerung teilweise recht deutlich unterscheiden, soll nun danach gefragt werden, wo in den jeweiligen Ländern die Statusgruppen zu verorten sind. Zur Erinnerung: Aktuelle Gegenwartsdiagnosen gehen davon aus, dass die Kritik an ökonomischer Ungleichheit eher ein Thema der unteren Statuslagen ist, die sich in Bezug auf neue Ungleichheitsfragen aber eher durch ,demarkationistische“ Haltungen, eine Skepsis gegenüber Diversität und ein weniger ausgeprägtes ökologisches Bewusstsein auszeichnen sollten. Für die oberen Statuslagen sollte sich hingegen ein umgekehrtes Einstellungsmuster zeigen. Finden sich diese sozialstrukturellen Profile nun in so diversen Ländern wie Schweden, Deutschland, Frankreich, Italien, Ungarn und Polen? Und wenn ja: Sind die Muster möglicher Einstellungsdifferenzen so ausgeprägt, dass man von polarisierten Orientierungen sprechen kann?

Um diese Frage zu beantworten, berechnen wir für jedes Land vier lineare Regressionsmodelle. Die abhängige Variable ist dabei jeweils einer der Einstellungsindizes. Als unabhängige Variable beziehen wir die Bildung, die Berufsklasse und die subjektive Einkommenslage (als vertikale Statusindikatoren) ein. Von Interesse sind für uns die auf der Basis dieser Regressionsanalysen vorhergesagten Einstellungswerte für unterschiedliche Statuslagen, wobei wir Statuslage über eine Kombination der vertikalen Indikatoren definieren. Da wir uns auf die theoretisch maximale zu erwartende Polarisierung konzentrieren und bei einer hohen Anzahl von Vergleichsdimensionen ein gewisses $\mathrm{Maß}$ an Übersichtlichkeit wahren möchten, nehmen wir nur zwei Statuslagen im Kontrast in den Blick: die höchste Statuslage (Kombination aus hoher Bildung, Berufsposition in der oberen Dienstklasse und der Einschätzung, dass der Haushalt bequem mit dem Einkommen leben kann) und die niedrigste Statuslage (Kombination aus niedriger Bildung, Berufsposition als einfacher Arbeiter und der Einschätzung, dass der Haushalt nur schwer oder sehr schwer mit dem Einkommen zurechtkommt). Es handelt sich damit um die sozialstrukturellen Pole, wodurch die Latte für die Zwei-Welten-Theorie eher niedrig gelegt wird.

Obgleich es keine klare Definition dafür gibt, ab wann man bei Einstellungsunterschieden von polarisierten Einstellungen sprechen kann, scheint es aus unserer Sicht plausibel, dann von statusmäßig polarisierten Einstellungen auszugehen, wenn es (a) Einstellungsunterschiede zwischen den Statuslagen gibt, (b) diese Unterschiede eine substanzielle Größe erreichen und (c) die Unterschiede qualitative Differenzen zwischen Zustimmung und Ablehnung implizieren (und nicht nur unterschiedliche Grade der Zustimmung bzw. Ablehnung anzeigen).

Wie stellen sich nun die Einstellungsunterschiede zwischen den Statusgruppen in den sechs Ländern dar? Die auf Basis der Regressionsanalysen vorhergesagten Einstellungswerte zeigen, dass in allen Ländern die unteren Statuslagen in der Tat kritischer auf ökonomische Ungleichheiten blicken als die oberen Statuslagen (Ab- 
bildung 3). ${ }^{14}$ Bei den neueren Ungleichheitsthemen verläuft die Strukturierung genau umgekehrt. Hier sind die Einstellungen zu Migration und sexuellen Minderheiten in den oberen Statuslagen offener bzw. anerkennungsbereiter als in den unteren Lagen, und auch die Aufmerksamkeit und Verantwortung gegenüber dem Klimawandel ist stärker ausgeprägt. Es fällt allerdings auch auf, dass man - zumindest auf der Basis der uns zur Verfügung stehenden Indikatoren - nur bei wenigen Themen eindeutig von einer statusmäßigen Polarisierung der Einstellungen sprechen kann. Dies ist am ehesten bei den migrationsbezogenen Einstellungen der Fall, die in vier der sechs betrachteten Länder (Deutschland, Frankreich, Italien und Polen) sehr deutliche Statusdifferenzen aufweisen, die zudem auch qualitative Unterschiede zwischen Zustimmung und Ablehnung anzeigen. Und es gilt auch für die Einstellungen zu sexueller Diversität in Italien. Neben diesen klaren Fällen finden sich auch Muster, bei denen es keine starken qualitativen Einstellungsunterschiede zwischen den Statusgruppen gibt, aber durchaus Unterschiede zwischen Zustimmung bzw. Ablehnung einerseits und eher indifferenten Haltungen andererseits. Dies gilt sowohl für die Einstellungen zu ökonomischer Ungleichheit (Polen) als auch für die Haltungen zu Migration (Schweden), sexueller Diversität (Polen und Ungarn) und Klimawandel (nahezu alle Länder).

In der Gesamtschau scheinen also am ehesten bei den „,neueren“ Ungleichheitsthemen statusmäßige Polarisierungen der Einstellungen vorzuliegen, und hier insbesondere beim Migrationsthema. Für die Themen Klimawandel und sexuelle Diversität finden wir eine solche Polarisierung nur in Ansätzen. Kaum polarisiert sind demgegenüber die Einstellungen beim klassischen Thema der ökonomischen Ungleichheit. Bei der Profilierung der Länder fallen vor allem Polen und Italien auf, wo statusmäßige Spaltungen oder Ansätze dazu in vielen Dimensionen existieren. In Schweden, Deutschland und Frankreich spielen solche Spaltungen - bis auf die Haltungen zu Migration - kaum eine Rolle. Diese empirischen Muster bleiben auch bestehen, wenn wir in erweiterten Regressionsmodellen nicht nur Bildung, Klasse und finanzielle Situation als unabhängige Variablen einbeziehen, sondern zusätzlich für Alter, Geschlecht und Urbanitätsgrad kontrollieren (ohne Abbildung; die entsprechenden Ergebnisse können bei den Autoren angefragt werden).

Wie stark ist aber nun diese statusmäßige Strukturierung im Vergleich zu anderen (horizontalen) Strukturgebern von Einstellungen? Dieser Frage soll im nächsten und letzten Schritt unserer Analysen nachgegangen werden. Bei der bisherigen Betrachtung der Einstellungsdifferenz zwischen oberen und unteren Statuslagen haben wir die mittleren Statuslagen sowie die absolute Größe der Statuslagen ausgeblendet. Es ist möglich, dass die Einstellungen zwischen oberen und unteren Lagen stark polarisiert sind, sich aber die meisten Menschen in einer mittleren Lage befinden, die Polarisierung also keinen insgesamt strukturierenden Einfluss hat. Eine wichtige ergänzende Perspektive zur Beantwortung der Frage, wie stark die vier Achsen der Ungleichheitseinstellungen sozialstrukturell verankert sind, bietet das statistische Maß der Bestimmtheit (adjustiertes $\mathrm{R}^{2}$ ). Dieses Maß gibt den Anteil der Varianz der abhängigen Variable an, der durch ein Set von unabhängigen Variablen im sta-

${ }^{14}$ Wir präsentieren hier nur die grafische Darstellung der Statusgruppenunterschiede. Die zugehörigen tabellarischen Ergebnisse der Regressionsanalysen sind auf Anfrage bei den Autoren erhältlich. 

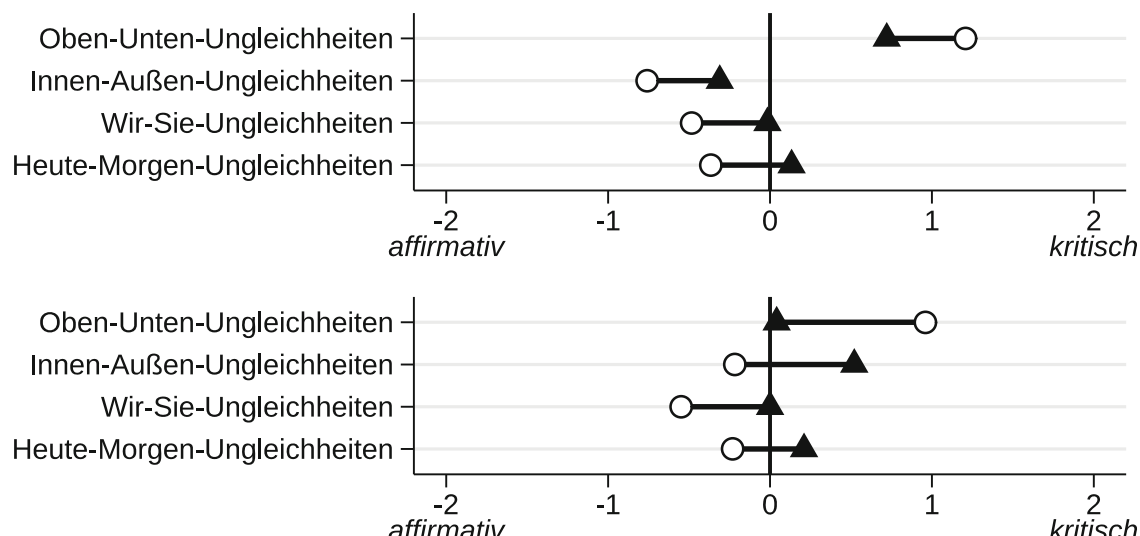

PL
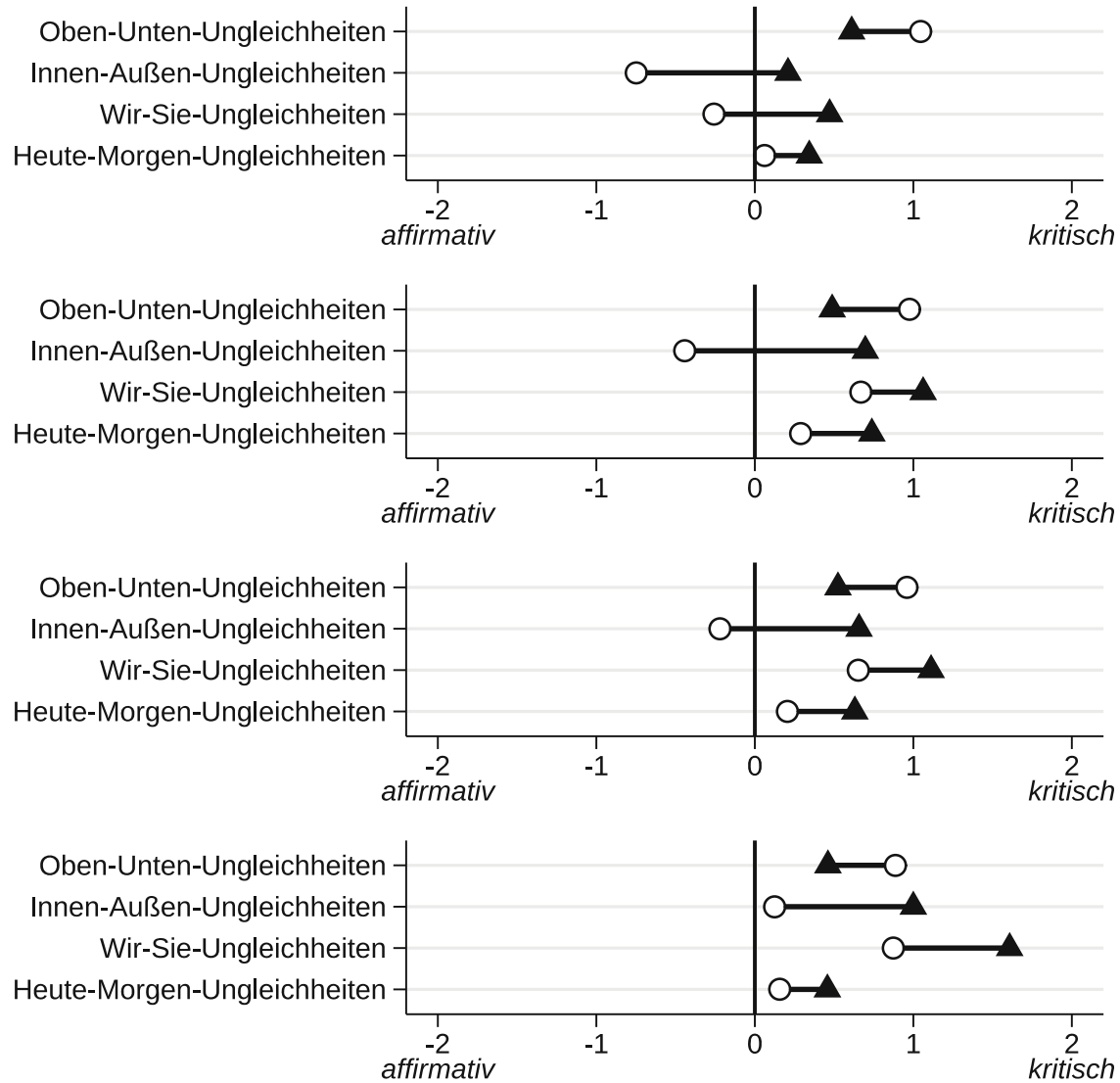

Statuslage $\boldsymbol{\Delta}$ hoch $\bigcirc$ niedrig

Abb. 3 Vorhergesagte Werte aus Regressionsmodellen für Personen in hoher Statuslage und Personen in niedriger Statuslage (Hohe Statuslage: Kombination aus hoher Bildung und guter subjektiver Einkommenslage und Tätigkeit in oberer Dienstklasse; Niedrige Statuslage: Kombination aus niedriger Bildung und schlechter subjektiver Einkommenslage und Tätigkeit als ungelernter Arbeiter) (Quelle: ESS 2016; eigene Berechnungen, gewichtet. Alle dargestellten länderspezifischen Einstellungsunterschiede zwischen den Statusgruppen sind signifikant auf dem 5\%-Niveau.) 
tistischen Sinn erklärt werden kann. Gegenüber Effektstärken bzw. vorhergesagten Werten ist dieses Maß zwar weniger direkt und eindeutig interpretierbar, es bietet aber den Vorteil, sowohl die Stärke der Effekte als auch die tatsächliche GruppengröBe der detaillierteren Statuslagen zu berücksichtigen. Für den Vergleich der Stärke von vertikalen und horizontalen Strukturgebern von Einstellungen werden wir nun dieses Bestimmtheitsmaß verwenden. Konkret haben wir für jedes Land und jede Dimension zwei Regressionsmodelle berechnet: In einem ersten Modell nehmen wir nur die vertikalen Statusindikatoren (Bildung, Berufsposition und Einkommen) als unabhängige Variable auf. Im zweiten Modell verwenden wir statt der vertikalen Statusindikatoren die horizontalen Charakteristika (Alter, Geschlecht, Urbanitätsgrad) als unabhängige Variablen.

Die in Tabelle 3 dargestellten Ergebnisse dieser Analysen zeigen, dass in allen Ländern der statusmäßig am stärksten polarisierte Einstellungskomplex, die Haltungen zur Migration, deutlich besser über die vertikalen Strukturgeber Klasse, Bildung und Einkommen erklärt werden kann als durch die horizontalen Merkmale Alter, Geschlecht und Urbanitätsgrad. Gleiches gilt für die Haltungen zu materieller Ungleichheit. Demgegenüber sind die Einstellungen zu sexuellen Minderheiten in fast allen Ländern (außer Ungarn) deutlich stärker über die horizontalen Merkmale strukturiert. Weiterführende Analysen, die den Beitrag jeder einzelnen Variable aufschlüsseln, zeigen, dass insbesondere das Alter eine entscheidende Rolle spielt (siehe Tabelle 7 im Anhang). Beim Klimawandel-Thema sind die Befunde gemischt: In den reicheren Ländern (Schweden, Frankreich, Deutschland) ist die Dimension stärker horizontal verankert, während in den weniger reichen Ländern (Ungarn, Polen und Italien) stärker die vertikalen Determinanten strukturierend wirken. Festgehalten werden sollte, dass die Varianzaufklärung der Modelle recht gering ist. Da aber solche Größenordnungen in der Einstellungsforschung nicht unüblich sind (zumal mit Modellen, die nur sozio-demographische Variablen enthalten und getrennt für horizontale und vertikale Merkmale geschätzt werden) und wir nicht an der maximalen Varianzaufklärung, sondern an der Vermessung der Strukturierung interessiert sind, fällt dies für unsere Untersuchung weniger ins Gewicht.

Insgesamt erkennen wir in Bezug auf die Einstellungsstrukturierung also ein hohes Maß an Gemeinsamkeiten zwischen den Ländern - mit stellenweisen Ausreißern. Kernbefund ist, dass die Haltungen zu klassischen Oben-Unten-Ungleichheiten in allen Ländern stärker vertikal als horizontal verankert sind. Bei den neueren Ungleichheitsthemen ist die Verankerung jedoch - je nach Thema, auf das man blickt - sehr unterschiedlich ausgeprägt: Während die Einstellungen zu Innen-Außen-Ungleichheiten einer vertikalen Strukturierung folgen, steht bei den Positionen zu Wir-Sie-Ungleichheiten eher eine horizontale Strukturierung im Vordergrund. Für die Heute-Morgen-Ungleichheiten kann wiederum kein klarer länderübergreifender Strukturierungsschwerpunkt festgestellt werden.

In Bezug auf unsere Forschungsfragen kann also Folgendes festgehalten werden: Erstens finden wir nur wenige Anhaltspunkte dafür, dass es sich bei den Einstellungen zu den aktuell besonders relevanten Ungleichheitsthemen um einen zweidimensionalen Einstellungsraum handelt, bei dem sich die Haltungen zu so diversen Aspekten wie Migration, sexueller Diversität und Klimaschutz zu einem umfassenderen soziokulturellen Einstellungskomplex bündeln, der dann lediglich von den 
Tab. 3 Strukturierung der Einstellungsdimensionen (adjustierte $\mathrm{R}^{2}$-Werte bivariater Regressionsmodelle) (Datenbasis: ESS 2016; eigene Berechnungen, gewichtet)

\begin{tabular}{|c|c|c|c|}
\hline Land & Ungleichheitsdimension & $\begin{array}{l}\text { Vertikale Statuslage } \\
\text { adj. } R^{2}\end{array}$ & $\begin{array}{l}\text { Horizontale Merkmale } \\
\text { adj. } R^{2}\end{array}$ \\
\hline \multirow[t]{4}{*}{$\mathrm{HU}$} & Oben-Unten & 3,89 & 1,93 \\
\hline & Innen-Außen & 4,53 & 1,24 \\
\hline & Wir-Sie & 2,71 & 1,99 \\
\hline & Heute-Morgen & 4,59 & 0,75 \\
\hline \multirow[t]{4}{*}{$\overline{\mathrm{PL}}$} & Oben-Unten & 9,26 & 4,13 \\
\hline & Innen-Außen & 4,36 & 1,30 \\
\hline & Wir-Sie & 3,02 & 5,84 \\
\hline & Heute-Morgen & 3,41 & 0,69 \\
\hline \multirow[t]{4}{*}{ IT } & Oben-Unten & 2,43 & 0,49 \\
\hline & Innen-Außen & 8,12 & 1,77 \\
\hline & Wir-Sie & 6,60 & 7,50 \\
\hline & Heute-Morgen & 2,67 & 0,51 \\
\hline \multirow[t]{4}{*}{$\overline{\mathrm{FR}}$} & Oben-Unten & 2,28 & 1,02 \\
\hline & Innen-Außen & 12,84 & 6,47 \\
\hline & Wir-Sie & 4,26 & 9,49 \\
\hline & Heute-Morgen & 2,42 & 4,88 \\
\hline \multirow[t]{4}{*}{$\mathrm{DE}$} & Oben-Unten & 2,47 & 0,46 \\
\hline & Innen-Außen & $\mathbf{7 , 8 4}$ & 2,77 \\
\hline & Wir-Sie & 3,16 & 10,13 \\
\hline & Heute-Morgen & 1,23 & 1,61 \\
\hline \multirow[t]{4}{*}{$\overline{\mathrm{SE}}$} & Oben-Unten & 4,41 & 3,59 \\
\hline & Innen-Außen & 12,25 & 5,95 \\
\hline & Wir-Sie & 6,59 & 12,37 \\
\hline & Heute-Morgen & 3,19 & 6,23 \\
\hline
\end{tabular}

Grundlage sind länder- und einstellungsspezifische lineare Regressionsmodelle, bei denen zum einen nur die vertikalen Charakteristika als unabhängige Variablen einbezogen wurden und zum anderen in separaten Modellen nur die horizontalen Charakteristika.

Horizontale Merkmale: Altersgruppen, Geschlecht, Urbanitätsgrad des Wohnortes.

Vertikale Statuslage: Klasse (5er-Schema), Bildung und subjektive ökonomische Lage.

Fett formatiert ist jeweils der größere Wert innerhalb einer länderspezifischen Ungleichheitsdimension.

Haltungen zu ökonomischer Ungleichheit abzugrenzen wäre. Viel eher besitzen auch die Einstellungen zu den neuen Ungleichheitsthemen eine gewisse Eigenständigkeit, der in empirischen Analysen Rechnung getragen werden sollte. Zweitens sind die oberen Statuslagen ungleichheitskritischer als die unteren Lagen, wenn es um die „neuen“ Ungleichheitsthemen geht. Beim klassischen Thema der ökonomischen Ungleichheit sind sie jedoch ungleichheitsaffiner. Dies ist ein Muster, das sich in allen Ländern unseres sehr heterogenen Samples zeigt. Allerdings erreichen diese Unterschiede nur bei wenigen Themen ein Ausmaß, das auf eine Polarisierung hindeutet. Dies ist am ehesten bei Migrationsfragen der Fall und in Ansätzen auch bei Fragen der Anerkennung von sexuellen Minderheiten. Ob eine solche Polarisierung vorliegt, ist allerdings hochgradig länderspezifisch und kann nicht als pauschales Muster generalisiert werden. Die empirische Tragfähigkeit der Annahme zweier gut abbildba- 
rer Lager kann anhand der hier vorgenommenen Operationalisierung jedenfalls nicht bestätigt werden. Drittens sind in allen Ländern die Einstellungen zu ökonomischer Ungleichheit stärker von den vertikalen Statusindikatoren Klasse, Bildung und Einkommen strukturiert als von den horizontalen Merkmalen Alter, Geschlecht und Urbanitätsgrad. Die neueren Ungleichheitsthemen besitzen demgegenüber, je nach Thema, recht unterschiedliche Strukturierungsschwerpunkte. Während für Haltungen zur Migration eher die vertikalen Merkmale im Vordergrund stehen, sind es bei Fragen der Anerkennung sexueller Minderheiten eher die horizontalen Charakteristika. Für das Thema der ökologischen Nachhaltigkeit lässt sich hingegen kein klares Strukturierungsmuster anhand der gewählten Merkmale ausmachen. Auch diese Befunde stehen Diagnosen einer relativ klaren, eindeutigen und zweidimensionalen sozialstrukturellen Ausprägung aktueller Konflikte entgegen.

\section{Diskussion}

Unser Artikel hat sich der aktuell vieldiskutierten Frage nach neuen gesellschaftlichen Konfliktlinien angenommen und Einstellungen zu sozialer Ungleichheit untersucht, wobei die Ungleichheitsfrage über die sozioökonomischen Aspekte hinaus auf Fragen der Inklusion, der Anerkennung und der Ökologie ausgeweitet und aufgefächert wurde. In diesen Feldern finden gegenwärtig politisierte Auseinandersetzungen statt, sei es darum, ob Migrantinnen und Migranten willkommen geheißen werden sollten oder nicht, ob Homosexualität toleriert und rechtlich gleichgestellt werden sollte oder nicht oder ob der Klimawandel und die Nachhaltigkeit als wichtig erachtet werden sollten oder nicht. All diese Problembereiche berühren wichtige Gerechtigkeits- und Anerkennungsfragen. Diese neuen Themenstellungen politischer Auseinandersetzung bilden sich in den öffentlichen Diskursen, in den Programmatiken politischer Parteien und in den Forderungskatalogen zivilgesellschaftlicher Akteure ab. In der Forschungsliteratur wird schon länger das Aufkommen neuer Konfliktlinien thematisiert, weil erwartet wird, dass sich hier veränderte, sozialstrukturell verankerte und manifeste Spaltungsstrukturen herausbilden könnten. Oftmals gehen diese Arbeiten von einer Spannung zwischen einem kosmopolitischen und kommunitaristischen Pol bzw. zwischen einem postmateriell-liberal-ökologischen und einem traditionell-autoritären Pol aus, aber es bleibt oft unklar, inwieweit sich damit auch „Realtypen“ verbinden, die entsprechende Einstellungsmuster aufweisen.

Um diese Frage zu beantworten, haben wir Einstellungsdaten zu unterschiedlichen Ungleichheitsfragen bemüht, von denen wir annehmen, dass sie mit einer möglichen Lagerbildung korrespondieren sollten. Diese haben wir als Oben-UntenUngleichheiten, Wir-Sie-Ungleichheiten, Innen-Außen-Ungleichheiten und HeuteMorgen-Ungleichheiten bezeichnet. Statt - wie ein Teil der Literatur zum Kosmopolitismus/Kommunitarismus-Schisma - von zusammenhängenden Einstellungssyndromen auszugehen, konnten wir zeigen, dass die vier Arenen der Ungleichheit relativ unabhängig voneinander zu betrachten sind. Die untersuchten europäischen Gesellschaften lassen sich vor dem Hintergrund der durchgeführten Analysen jedenfalls nicht unisono und holzschnittartig als Konfliktarenen der Kosmopoliten und Kommunitaristen bezeichnen. Zwar zeigt sich in den Faktorenanalysen eine schwa- 
che Mehrfachladung einzelner Items, die einen gewissen Zusammenhang zwischen den ,neuen“ Konflikten um Migration und sexuelle Diversität anzeigen, aber dieser Zusammenhang ist, erstens, nicht besonders stark ausgeprägt, und zweitens unterscheiden sich die Dimensionen auch in ihrer sozialstrukturellen Verankerung. Der Innen-Außen-Konflikt ist sehr viel stärker ökonomisch geprägt, während beim WirSie-Konflikt horizontale Merkmale relevanter sind. Alle Konflikte zeigen aber zu einem gewissen Grad die erwartete soziale Strukturierung: Die unteren Statuslagen kritisieren ökonomische Ungleichheit stärker, die oberen Statuslagen sind bei den neuen Konfliktthemen progressiver. Besonders stark ist diese Spaltung aber nur beim Migrationsthema. Länderunterschiede finden sich vor allem in der Aushandlung der neuen Konflikte, allerdings nur sehr eingeschränkt beim Klimakonflikt. Die vergleichsweise geringen Unterschiede bei den Oben-Unten-Ungleichheiten sind auf die Konsensfähigkeit eines gewissen Maßes an Sozialstaatlichkeit überall in Europa zurückzuführen.

Es wäre aber falsch zu folgern, dass es generell keine Konflikte um eine umfassendere redistributive Sozialpolitik, wie beispielsweise höhere Vermögens- oder Spitzensteuersätze, gibt. Um solche ökonomischen Interessengegensätze einzufangen, wären jedoch Items zu detaillierteren Themen der materiellen Ungleichheit und Verteilung notwendig, die in den von uns verwendeten Daten nicht zur Verfügung standen. Bei den Einstellungen zu den neueren Ungleichheitsthemen gehen wir ebenfalls davon aus, dass wir mit unseren Items zwar wichtige, aber natürlich nicht alle Aspekte der gegenwärtigen Auseinandersetzungen abbilden: Bei den Wir-Sie-Ungleichheiten beziehen wir u.a. das Adoptionsrecht für Homosexuelle ein, das häufig im Zentrum medialer und gesellschaftlicher Diskussionen stand. Zudem wissen wir aus vorangegangen Studien (Mau et al. 2020), dass Items zur Akzeptanz von Homosexualität sehr hoch mit der Anerkennung von Transgender-Personen korrelieren, einem weiteren aktuell oft diskutierten Thema. Mit spezifischeren Konfliktthemen - zum Beispiel zur inklusiven Sprache - wäre es aber möglich, dass sich auch Einstellungen stärker auskonturieren. Bei den Innen-Außen-Ungleichheiten haben wir unseren Blick auf die subjektiv eingeschätzten ökonomischen und kulturellen Effekte von Migration gerichtet und bei den Heute-Morgen-Ungleichheiten u.a. auf die persönliche Verantwortung im Kampf gegen den Klimawandel. Auch dies sind Aspekte, die sich in den aktuellen Debatten um neuere Ungleichheitsthemen wiederfinden und dort zentrale Punkte berühren. Dennoch könnten auch bei diesen neueren Ungleichheitsthemen weitere (Unter-)Dimensionen sichtbar werden, wenn mehr und detailliertere Items in die Untersuchung einbezogen würden. Dies würde allerdings unserem Befund, dass der zeitgenössische Konfliktraum mehr als zwei Dimensionen aufweist, nicht notwendigerweise widersprechen, sondern ihn eher detaillierter ausbuchstabieren. Darüber hinaus muss darauf hingewiesen werden, dass sich unsere Analysen auf den Zeitraum von August 2016 bis November 2017 beziehen und damit auf den Vorabend der Gründung der Fridays-for-Future-Bewegung, die einen starken Mobilisierungs- und Politisierungsschub für das Klimawandel-Thema mit sich brachte. Insofern kann es möglich sein, dass wir den aktuellen Grad der Polarisierung in dieser Dimension tendenziell unterschätzen. Für die anderen Arenen der Ungleichheit ist jedoch eine solche, unsere Ergebnisse tangierende Dynamik kaum erkennbar. Insbesondere der ,lange Sommer der Migration“ (Hess et al. 2017) 
des Jahres 2015 lag deutlich vor dem Erhebungszeitpunkt unserer Daten. Um eine genauere Einschätzung der Stabilität bzw. Dynamik des Konfliktgeschehens treffen zu können, sollten zukünftige Untersuchungen aktuellere Daten heranziehen und möglichst mit einem erweiterten Set an Items arbeiten.

Trotz dieser kleineren Einschränkungen zeigt unsere Studie, dass die recht starke Polarisierung, die wir mitunter in der Parteienlandschaft und den medialen Diskursen finden können, in den Einstellungen nicht in der gleichen Stärke gespiegelt wird. Oftmals finden sich moderate Formen der Zustimmung zu den Items; die Polarisierung der Einstellungen ist - mit Ausnahme der Migrationsfrage - nicht sonderlich stark. Zudem lassen die sozialstrukturellen Determinanten zwar erkennen, dass es eine Differenzierung der Einstellungsprofile über die Bevölkerungen hinweg gibt, aber auch sie ist nicht sehr deutlich ausgeprägt. Bei allen Items finden wir einen sozialen Gradienten, aber keine separaten oder auskonturierten Lager, sodass man wohl davon ausgehen muss, dass die Unterscheidung zwischen Kosmopoliten und Kommunitaristen eher auf Idealtypen, denn auf Realtypen verweist (Zürn 2020). Konfliktfelder mögen sich verschieben - ob sich damit schon eine klar erkennbare Cleavage-Struktur ergibt, steht auf einem anderen Blatt. Unsere Analysen liefern jedenfalls nur überschaubare Hinweise darauf, dass hier ein Gegeneinander sozialstrukturell wie einstellungsmäßig disparater gesellschaftlicher Gruppen entsteht. Neuere Forschungen verweisen zudem darauf, dass die Ausprägung von Einstellungsdifferenzen (und womöglich von Spaltungsstrukturen) mit Formen der gesellschaftlichen Politisierung bestimmter Themen zu tun haben könnte (Ares 2021). Inwieweit ein Issue umkämpft ist und in welcher Form Polarisierung auffindbar ist, hätte dann eher etwas mit parteipolitischen Auseinandersetzungen zu tun denn mit vorgängigen sozialstrukturellen Cleavages. Nicht sozialstrukturelle Polarisierung wäre dann verantwortlich für die Politisierung, sondern die Politisierung selbst wäre ein Katalysator der Polarisierung. 


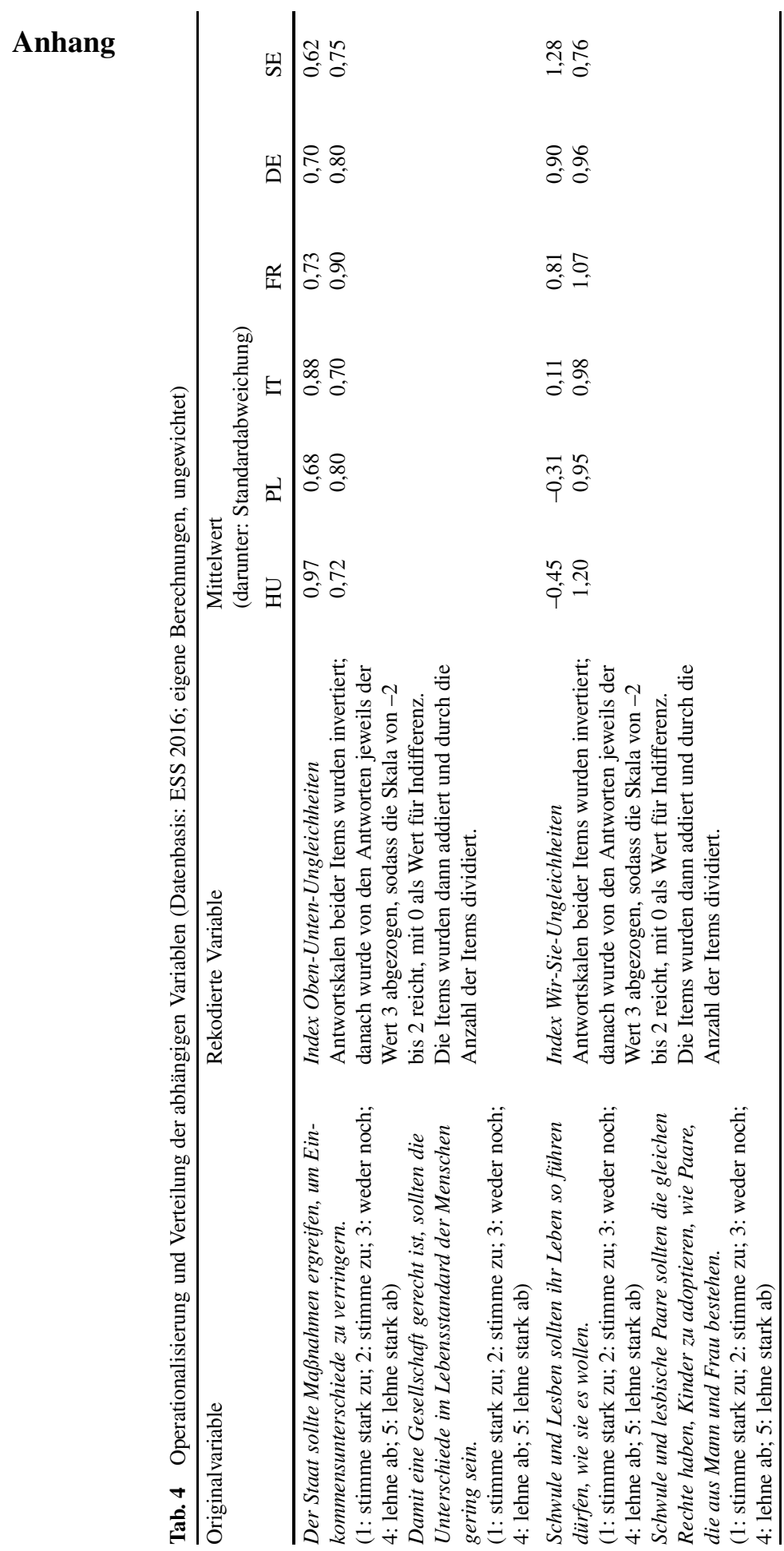




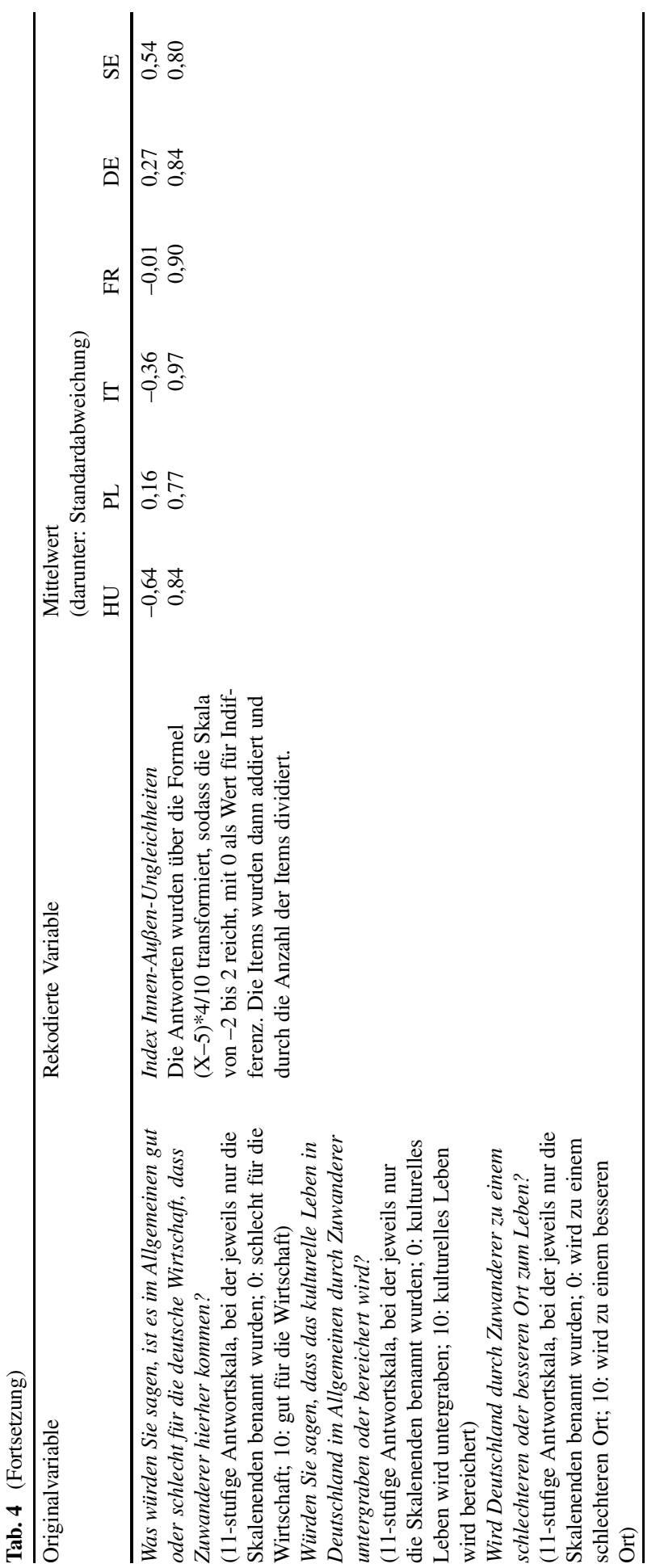




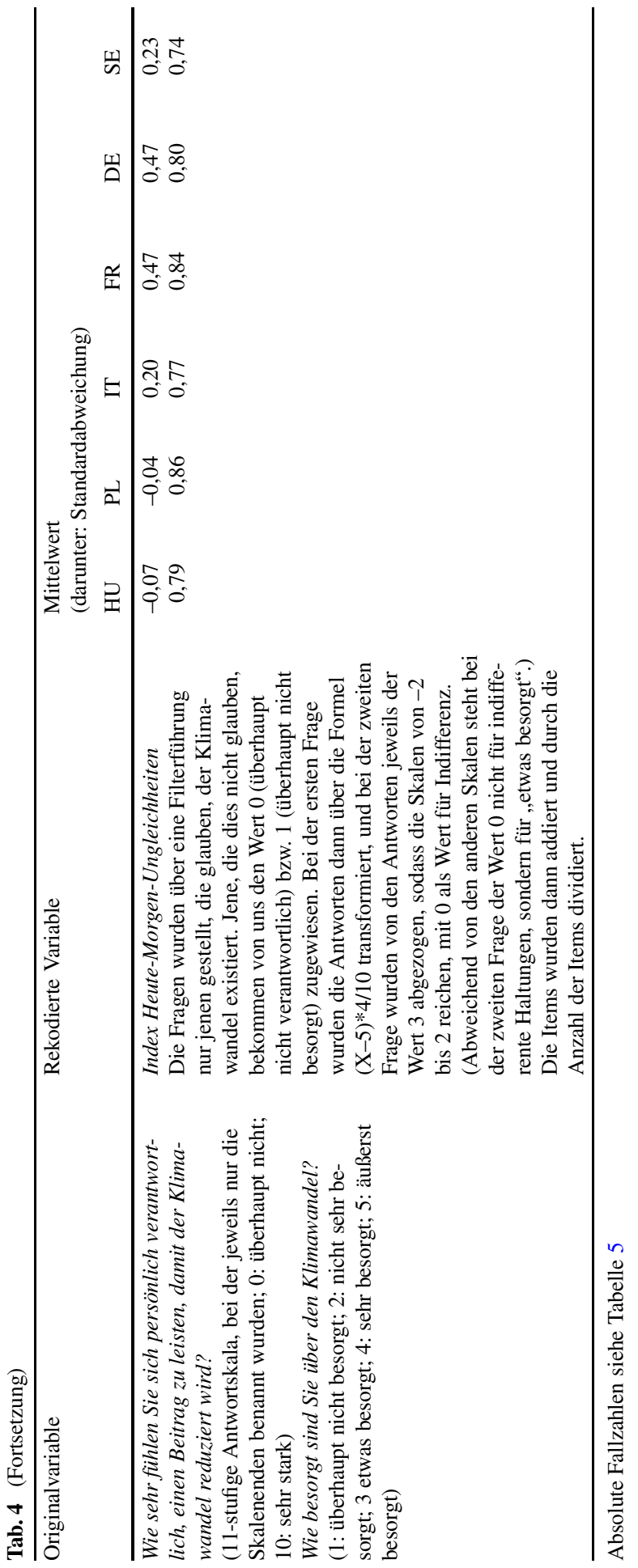




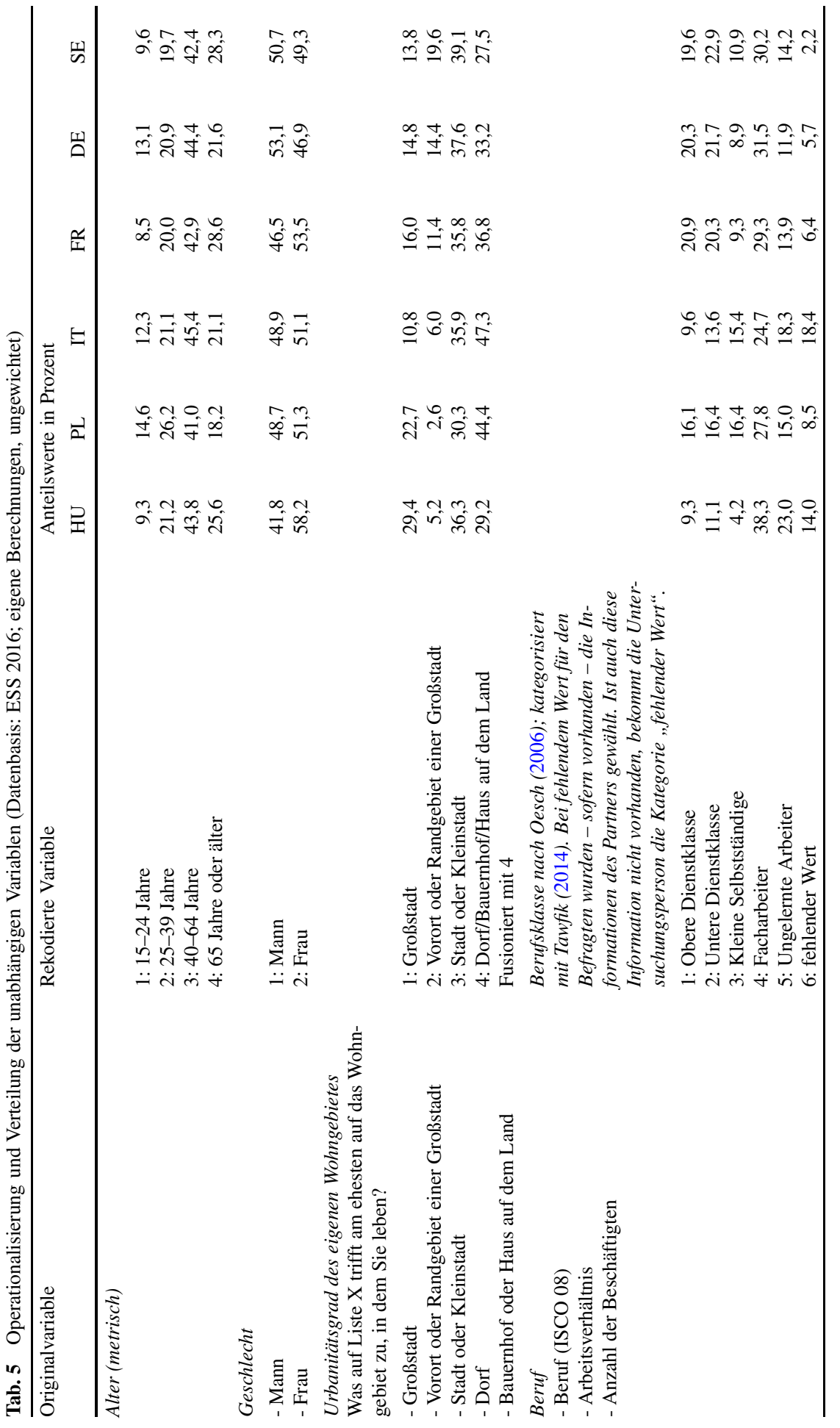




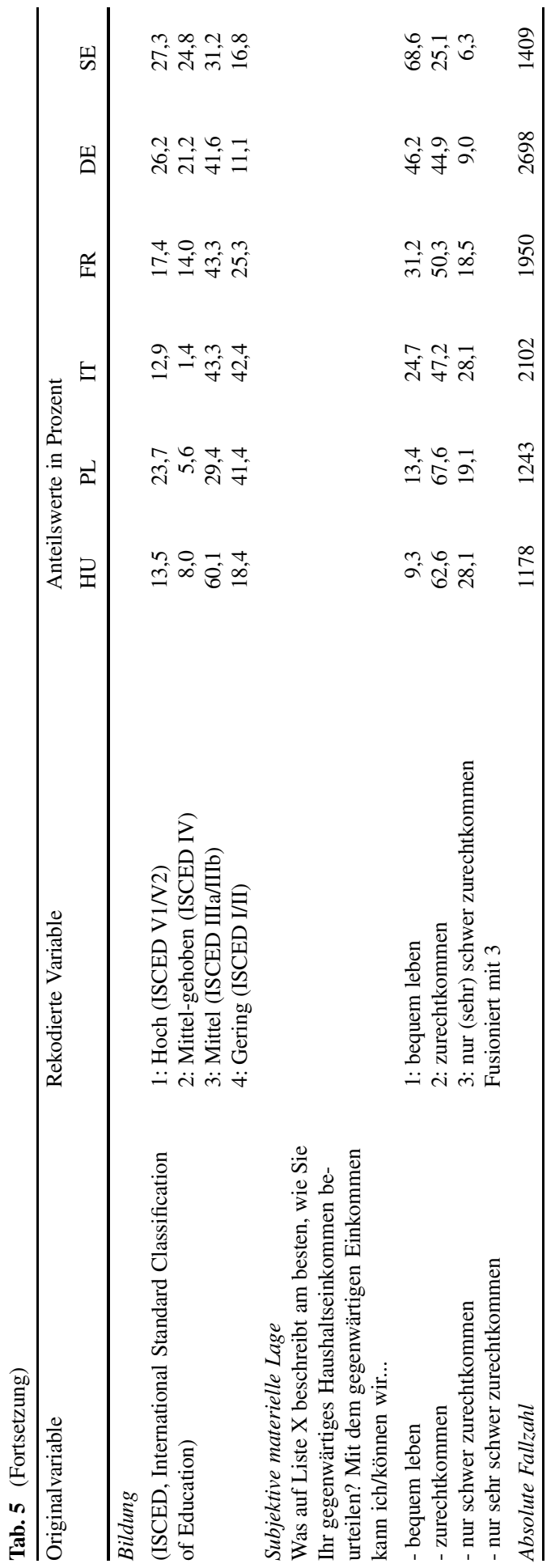




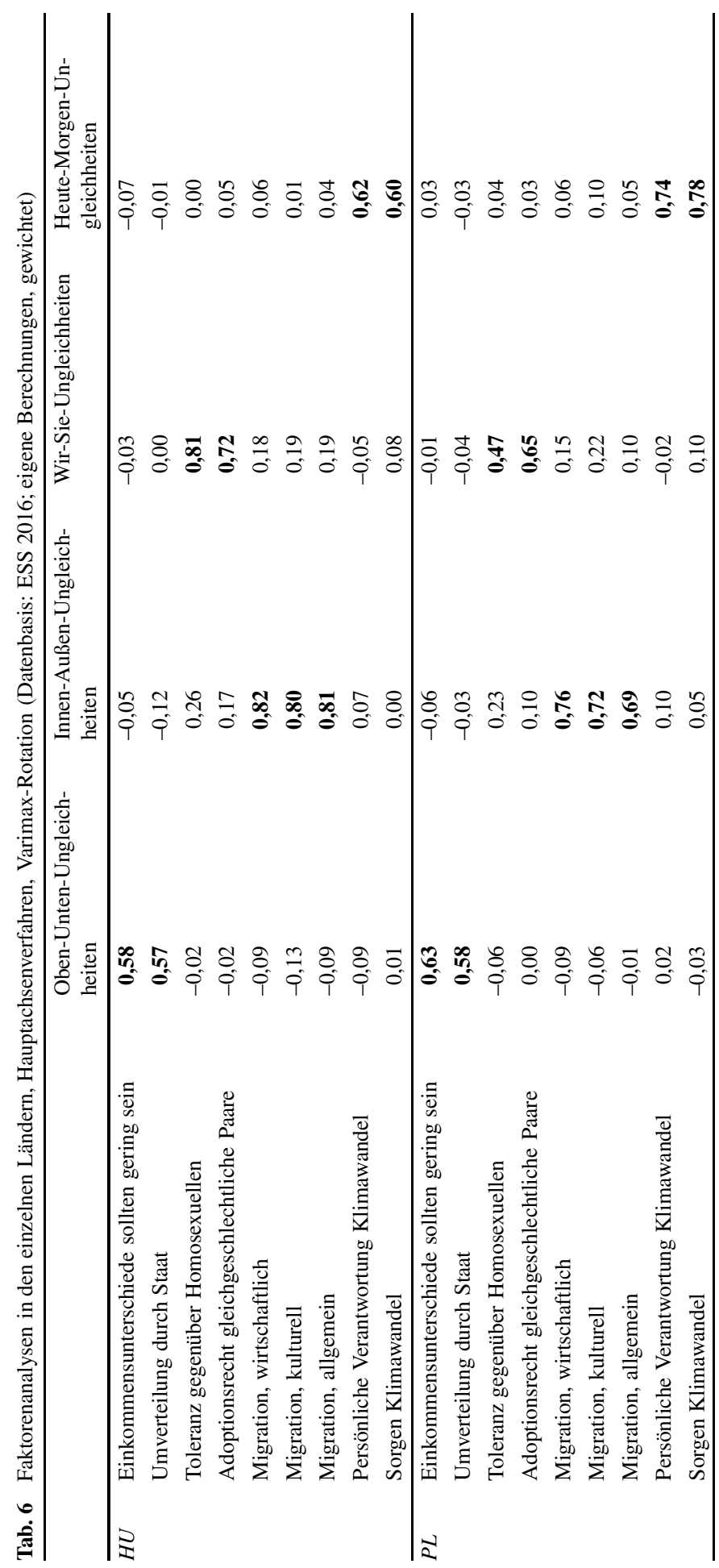




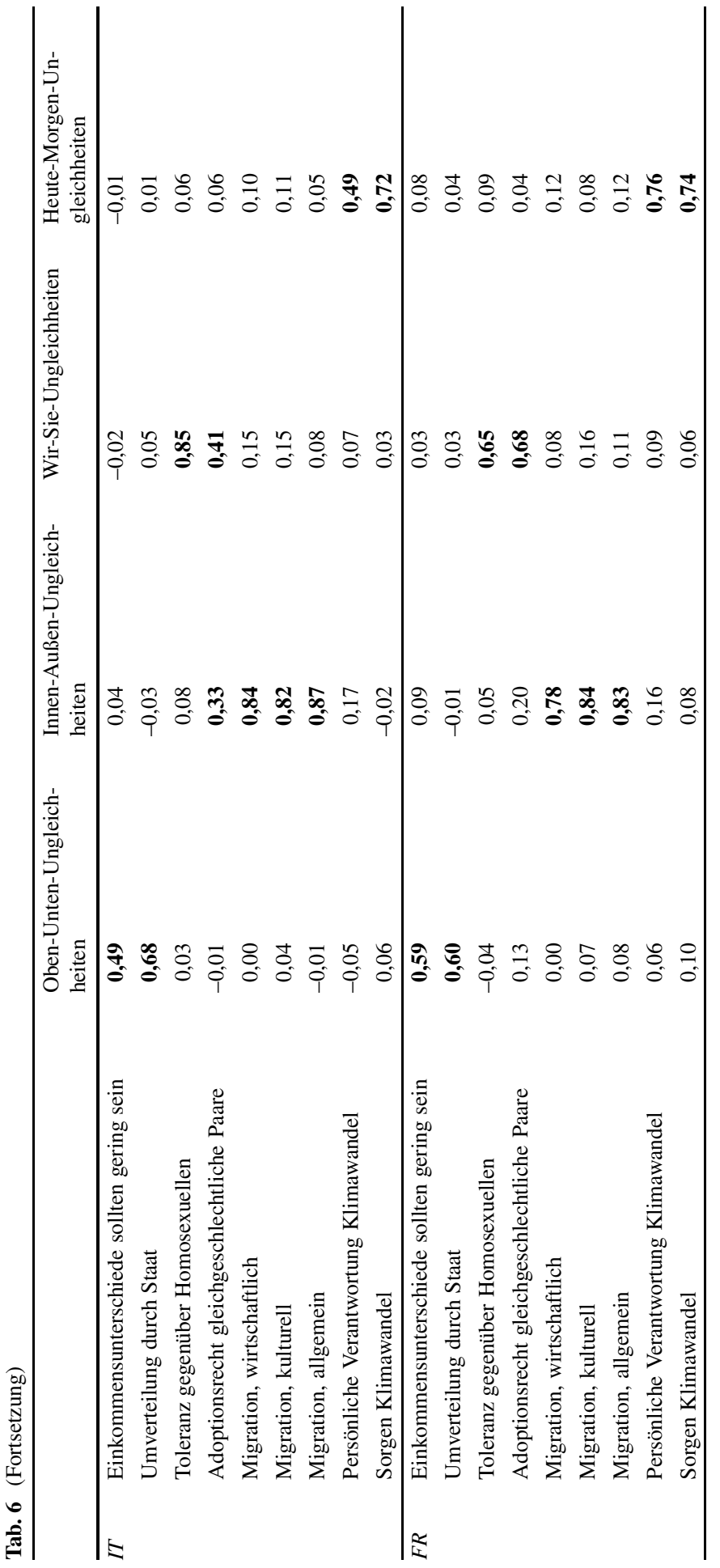




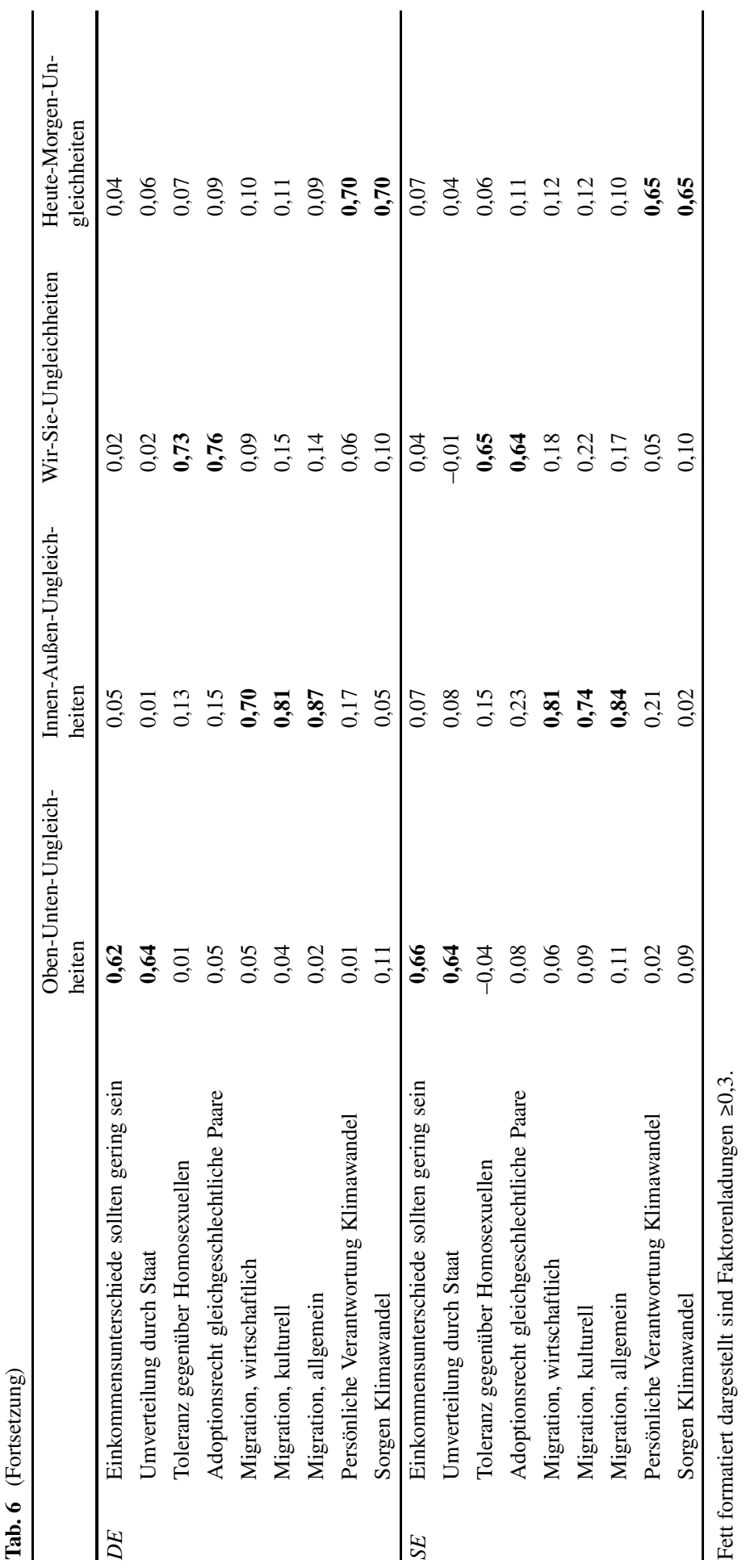


Tab. 7 Adjustierte $\mathrm{R}^{2}$-Werte für die bivariaten Zusammenhänge zwischen den Dimensionen und den einzelnen unabhängigen Variablen in den jeweiligen Ländern (Datenbasis: ESS 2016; eigene Berechnungen, gewichtet)

\begin{tabular}{|c|c|c|c|c|c|c|c|}
\hline Land & Ungleichheitsdimension & Alter & Geschlecht & Urbanität & Klasse & Einkommen & Bildung \\
\hline \multirow[t]{4}{*}{$H U$} & Oben-Unten & $-0,16$ & $-0,01$ & 2,13 & 3,18 & 1,18 & 2,28 \\
\hline & Innen-Außen & 0,16 & 0,13 & 1,05 & 2,35 & 0,63 & 3,97 \\
\hline & Wir-Sie & 0,59 & $-0,06$ & 1,53 & 1,20 & 1,30 & 1,40 \\
\hline & Heute-Morgen & 0,16 & 0,32 & 0,28 & 0,77 & 1,87 & 3,51 \\
\hline \multirow[t]{4}{*}{$P L$} & Oben-Unten & 0,48 & $-0,08$ & 3,58 & 3,64 & 4,20 & 6,72 \\
\hline & Innen-Außen & 0,29 & $-0,08$ & 0,96 & 2,12 & 2,42 & 2,94 \\
\hline & Wir-Sie & 1,55 & 1,49 & 2,40 & 0,94 & 1,06 & 2,48 \\
\hline & Heute-Morgen & 0,22 & 0,07 & 0,32 & 1,98 & 0,25 & 3,20 \\
\hline \multirow[t]{4}{*}{$I T$} & Oben-Unten & 0,31 & 0,17 & 0,03 & 1,46 & 1,58 & 1,27 \\
\hline & Innen-Außen & 1,30 & 0,07 & 0,30 & 3,08 & 4,73 & 5,19 \\
\hline & Wir-Sie & 7,24 & 0,22 & $-0,03$ & 2,01 & 2,07 & 5,44 \\
\hline & Heute-Morgen & 0,64 & $-0,04$ & $-0,10$ & 1,35 & 0,33 & 2,40 \\
\hline \multirow[t]{4}{*}{$F R$} & Oben-Unten & 0,19 & 0,68 & 0,13 & 0,40 & 1,55 & 0,93 \\
\hline & Innen-Außen & 2,39 & 0,15 & 4,75 & 1,52 & 5,40 & 9,19 \\
\hline & Wir-Sie & 8,15 & 0,91 & 0,10 & 1,10 & 1,54 & 1,67 \\
\hline & Heute-Morgen & 3,96 & $-0,01$ & 0,97 & 0,32 & 0,52 & 2,19 \\
\hline \multirow[t]{4}{*}{$\overline{D E}$} & Oben-Unten & 0,16 & 0,01 & 0,29 & 1,07 & 1,73 & 0,29 \\
\hline & Innen-Außen & 0,45 & 0,29 & 2,05 & 4,09 & 3,87 & 4,19 \\
\hline & Wir-Sie & 6,68 & 2,45 & 0,68 & 1,19 & 2,06 & 1,30 \\
\hline & Heute-Morgen & 0,89 & 0,39 & 0,26 & 0,83 & 0,42 & 0,93 \\
\hline \multirow[t]{4}{*}{$S E$} & Oben-Unten & 1,80 & 1,40 & 0,60 & 3,11 & 1,77 & $-0,13$ \\
\hline & Innen-Außen & 1,17 & 1,06 & 4,23 & 8,70 & 1,08 & 9,09 \\
\hline & Wir-Sie & 7,55 & 2,97 & 2,83 & 3,40 & 1,23 & 4,00 \\
\hline & Heute-Morgen & 2,52 & 2,93 & 0,83 & 2,21 & 0,19 & 2,33 \\
\hline
\end{tabular}

Für jede Zelle wurde ein dimensions- und länderspezifisches Regressionsmodell berechnet, in das jeweils nur ein Merkmal der Befragungsperson (Alter, Geschlecht, Urbanitätsgrad, Klasse, subjektives Einkommen, Bildung) als unabhängige Variable aufgenommen wurde.

Funding Open Access funding enabled and organized by Projekt DEAL.

Open Access Dieser Artikel wird unter der Creative Commons Namensnennung 4.0 International Lizenz veröffentlicht, welche die Nutzung, Vervielfältigung, Bearbeitung, Verbreitung und Wiedergabe in jeglichem Medium und Format erlaubt, sofern Sie den/die ursprünglichen Autor(en) und die Quelle ordnungsgemäß nennen, einen Link zur Creative Commons Lizenz beifügen und angeben, ob Änderungen vorgenommen wurden.

Die in diesem Artikel enthaltenen Bilder und sonstiges Drittmaterial unterliegen ebenfalls der genannten Creative Commons Lizenz, sofern sich aus der Abbildungslegende nichts anderes ergibt. Sofern das betreffende Material nicht unter der genannten Creative Commons Lizenz steht und die betreffende Handlung nicht nach gesetzlichen Vorschriften erlaubt ist, ist für die oben aufgeführten Weiterverwendungen des Materials die Einwilligung des jeweiligen Rechteinhabers einzuholen.

Weitere Details zur Lizenz entnehmen Sie bitte der Lizenzinformation auf http://creativecommons.org/ licenses/by/4.0/deed.de. 


\section{Literatur}

van den Akker, H., van der Ploeg, R., \& Scheepers, P. (2013). Disapproval of homosexuality: Comparative research on individual and national determinants of disapproval of homosexuality in 20 European countries. International Journal of Public Opinion Research, 25, 64-86.

Andersen, R., \& Fetner, T. (2008). Economic inequality and intolerance: Attitudes toward homosexuality in 35 democracies. American Journal of Political Science, 52, 942-958.

Ares, M. (2021). Issue politization and social class: How the electoral supply activates class divides in political preferences. European Journal of Political Research (online first). https://doi.org/10.1111/ 1475-6765.12469.

Arts, W., \& Gijsberts, M. (1998). After the velvet revolutions: Altered life-chances, fragile legitimacy, and split-consciousness in post-communist Eastern Europe. Social Justice Research, 11, 143-171.

Bartholomew, D. J., Steele, F., \& Moustaki, I. (2008). Analysis of multivariate social science data. Boca Raton: CRC press.

Biskamp, F. (2020). Die Arbeiter_Innen, der Autoritarismus und die AfD. Einige Daten aus Runde 9 des European Social Survey. http://blog.florisbiskamp.com/2020/02/02/die-arbeiter_innen-derautoritarismus-und-die-afd/. Zugegriffen: Dez. 2020.

Bornschier, S. (2010). Cleavage politics and the populist right. The new cultural conflict in Western Europe. Philadelphia: Temple University Press.

Brooks, C., \& Manza, J. (2008). Why welfare states persist. The importance of public opinion in democracies. Chicago: University of Chicago Press.

Burck, J., Marten, F., Bals, C., \& Höhne, N. (2017). Klimaschutz-Index. Die wichtigsten Ergebnisse 2018. Berlin, Köln: New Climate Institute.

Chaisty, P., \& Whitefield, S. (2015). Attitudes towards the environment: Are post-communist societies (still) different? Environmental Politics, 24, 598-616.

De Wilde, P., Koopmans, R., Merkel, W., \& Zürn, M. (2019). The struggle over borders: Cosmopolitanism and communitarianism. Cambridge: Cambridge University Press.

Dotti Sani, G. M., \& Quaranta, M. (2020). Let them be, not adopt: General attitudes towards gays and lesbians and specific attitudes towards adoption by same-sex couples in 22 European countries. Social Indicators Research, 150, 351-373.

van Dyk, S. (2019). Identitätspolitik gegen ihre Kritik gelesen. Für einen rebellischen Universalismus. Aus Politik und Zeitgeschichte, 69(9-11), 25-32.

Eick, G. M. (2020). Education as liberation? A critical analysis of the relationship between higher education and welfare chauvinism across Europe. Dissertation. Canterbury: University of Kent.

ESS (2016). European Social Survey Round 8 data. Data file edition 2.2. NSD - Norwegian Centre for Research Data, Norway - Data Archive and distributor of ESS data for ESS ERIC. https://doi.org/10. 21338/NSD-ESS8-2016.

Europäische Kommission (2008). Europeans' attitudes towards climate change. Eurobarometer 69. https:// ec.europa.eu/commfrontoffice/publicopinion/archives/ebs/ebs_300_full_en.pdf. Zugegriffen: Dez. 2020.

European Social Survey (2018). ESS8 - 2016 documentation report. The ESS data archive edition 2.1. Norwegian Centre for Research Data. https://www.europeansocialsurvey.org/docs/round8/survey/ESS8_ data_documentation_report_e02_1.pdf. Zugegriffen: Dez. 2020.

Evans, M. D., \& Kelley, J. (2018). Strong welfare states do not intensify public support for income redistribution, but even reduce it among the prosperous: A multilevel analysis of public opinion in 30 countries. Societies, 8(4), Art. 105.

Fitzgerald, R., Winstone, L., \& Prestage, Y. (2014). Searching for evidence of acculturation: Attitudes toward homosexuality among migrants moving from Eastern to Western Europe. International Journal of Public Opinion Research, 26, 323-341.

Flores, A., \& Park, A. (2018). Polarized progress. Social acceptance of LGBT people in 141 countries 1981 to 2014. Los Angeles: The Williams Institute/UCLA School of Law. https://williamsinstitute. law.ucla.edu/wp-content/uploads/Polarized-Progress-GAI-Mar-2018.pdf. Zugegriffen: Dez. 2020.

Ford, R., \& Jennings, W. (2020). The changing cleavage politics of Western Europe. Annual Review of Political Science, 23, 295-314.

Foroutan, N. (2019). Die postmigrantische Gesellschaft. Ein Versprechen der pluralen Demokratie. Bielefeld: transcript.

Franzen, A., \& Vogl, D. (2013). Two decades of measuring environmental attitudes: A comparative analysis of 33 countries. Global Environmental Change, 23, 1001-1008. 
Fraser, N. (2017). From progressive neoliberalism to Trump - and beyond. American Affairs, 1(4), 46-64.

Fraser, N., \& Honneth, A. (2003). Umverteilung oder Anerkennung? Eine politisch-philosophische Kontroverse. Frankfurt a. M.: Suhrkamp.

Fritz, M., \& Koch, M. (2019). Public support for sustainable welfare compared: Links between attitudes towards climate and welfare policies. Sustainability, 11(15), Art. 4146.

Fukuyama, F. (2019). Identität. Wie der Verlust der Würde unsere Demokratie gefährdet. Hamburg: Hoffmann und Campe.

Gerhards, J. (2010). Non-discrimination towards homosexuality: The European Union's policy and citizens' attitudes towards homosexuality in 27 European countries. International Sociology, 25, 5-28.

Gidron, N., \& Hall, P. A. (2017). The politics of social status: Economic and cultural roots of the populist right. The British Journal of Sociology, 68(S1), 57-84.

Heath, A. F., \& Richards, L. (2020). Contested boundaries: Consensus and dissensus in European attitudes to immigration. Journal of Ethnic and Migration Studies, 46, 489-511.

Heisterhagen, N. (2018). Die liberale Illusion. Warum wir einen linken Realismus brauchen. Bonn: Dietz.

Hess, S., Kasparek, B., Kron, S., Rodatz, M., Schwertl, M., \& Sontowski, S. (2017). Der lange Sommer der Migration. Berlin: Assoziation A.

Horn, J. L. (1965). A rationale and test for the number of factors in factor analysis. Psychometrika, 30, $179-185$.

Jakobsson, N., Muttarak, R., \& Schoyen, M. A. (2018). Dividing the pie in the eco-social state: Exploring the relationship between public support for environmental and welfare policies. Environment and Planning C: Politics and Space, 36, 313-339.

Kaminska, O. (2020). Guide to using weights and sample design indicators with ESS data. https://www. europeansocialsurvey.org/docs/methodology/ESS_weighting_data_1_1.pdf. Zugegriffen: Dez. 2020.

Kluegel, J. R., \& Smith, E. R. (1986). Beliefs about inequality. Americans' views of what is and what ought to be. New York: Aldine de Gruyter.

Koppetsch, C. (2019). Die Gesellschaft des Zorns. Rechtspopulismus im globalen Zeitalter. Bielefeld: transcript.

Kriesi, H., \& Grande, E. (2004). Nationaler politischer Wandel in entgrenzten Räumen. In U. Beck \& C. Lau (Hrsg.), Entgrenzung und Entscheidung. Was ist neu an der Theorie reflexiver Modernisierung? (S. 402-429) Frankfurt a. M.: Suhrkamp.

Kriesi, H., Grande, E., Dolezal, M., Helbling, M., Höglinger, D., Hutter, S., et al. (2012). Political conflict in Western Europe. Cambridge: Cambridge University Press.

Kriesi, H., Grande, E., Lachat, R., Dolezal, M., Bornschier, S., \& Frey, T. (2008). West European politics in the age of globalization. Cambridge: Cambridge University Press.

Kurer, T., \& Palier, B. (2019). Shrinking and shouting: The political revolt of the declining middle in times of employment polarization. Research \& Politics, 6, 1-6.

Lilla, M. (2018). The once and future liberal: After identity politics. Oxford: Oxford University Press.

Lindh, A., \& McCall, L. (2020). Class position and political opinion in rich democracies. Annual Review of Sociology, 46, 419-441.

Lipset, S. M., \& Rokkan, S. (1967). Cleavage structures, party systems, and voter alignments: An introduction. New York: Free Press.

Lux, T. (2011). Jenseits sozialer Klassen? Eine empirische Überprüfung der Individualisierungsthese am Beispiel von Ungleichheitseinstellungen und Wahlverhalten. Zeitschrift für Soziologie, 40, 436-457.

Marks, G., Wilson, C. J., \& Ray, L. (2002). National political parties and European integration. American Journal of Political Science, 46, 585-594.

Mau, S. (2003). Moral economy of welfare states. Britain and Germany compared. London: Routledge.

Mau, S., \& Mewes, J. (2012). Unraveling working-class welfare chauvinism. In S. Svallfors (Hrsg.), Contested welfare states: Welfare attitudes in Europe and beyond. Stanford: Stanford University Press.

Mau, S., Lux, T., \& Gülzau, F. (2020). Die drei Arenen der neuen Ungleichheitskonflikte. Eine sozialstrukturelle Positionsbestimmung der Einstellungen zu Umverteilung, Migration und sexueller Diversität. Berliner Journal für Soziologie, 30, 317-346.

Merkel, W. (2017a). Kosmopolitismus versus Kommunitarismus: Ein neuer Konflikt in der Demokratie. In P. Harfst, I. Kubbe \& T. Poguntke (Hrsg.), Parties, governments and elites: The comparative study of democracy (S. 9-23). Wiesbaden: Springer VS.

Merkel, W. (2017b). Die populistische Revolte. Kulturpolitische Mitteilungen, (157), 53-56.

Merkel, W. (2021). Polarisierung als gesellschaftliche Signatur. WZB-Mitteilungen. https://www.wzb.eu/ de/publikationen/wzb-mitteilungen/polarisierung-und-gesellschaft/polarisierung-als-gesellschaftlichesignatur. Zugegriffen: Aug. 2021.

MIPEX (2015). Migrant integration policy index 2015. Brussels: Migrant Policy Group. 
Neckel, S. (2018). Ökologische Distinktion. Soziale Grenzziehung im Zeichen von Nachhaltigkeit. In S. Neckel, N. Besedovsky, M. Boddenberg, M. Hasenfratz, S. M. Pritz \& T. Wiegand (Hrsg.), Die Gesellschaft der Nachhaltigkeit. Umrisse eines Forschungsprogramms (S. 59-76). Bielefeld: transcript.

Neckel, S. (2020). Der Streit um die Lebensführung. Nachhaltigkeit als sozialer Konflikt. Mittelweg 36, 29(6), 82-100.

Norris, P., \& Inglehart, R. (2019). Cultural backlash: Trump, Brexit, and authoritarian populism. Cambridge: Cambridge University Press.

OECD (2020). Income distribution database. https://stats.oecd.org/Index.aspx?DataSetCode=IDD. Zugegriffen: Dez. 2020.

Oesch, D. (2006). Redrawing the class map. Stratification and institutions in Britain, Germany, Sweden and Switzerland. Basingstoke: Palgrave Macmillan.

Oesch, D., \& Rennwald, L. (2018). Electoral competition in Europe's new tripolar political space: Class voting for the left, centre-right and radical right. European Journal of Political Research, 57, 783-807.

Otto, A., \& Gugushvili, D. (2020). Eco-social divides in Europe: Public attitudes towards welfare and climate change policies. Sustainability, 12(1), Art. 404.

Pittau, M. G., Massari, R., \& Zelli, R. (2013). Hierarchical modelling of disparities in preferences for redistribution. Oxford Bulletin of Economics and Statistics, 75(4), 556-584.

Proto, E., \& Rustichini, A. (2012). Life satisfaction, household income and personality traits. Working paper no. 86. Coventry: Department of Economics, University of Warwick.

Reckwitz, A. (2017). Die Gesellschaft der Singularitäten. Zum Strukturwandel der Moderne. Berlin: Suhrkamp.

Reckwitz, A. (2019). Das Ende der Illusionen. Politik, Ökonomie und Kultur in der Spätmoderne. Berlin: Suhrkamp.

Röder, A., \& Lubbers, M. (2015). Attitudes towards homosexuality amongst recent Polish migrants in Western Europe: Migrant selectivity and attitude change. Journal of Ethnic and Migration Studies, $41,1858-1884$.

Roosma, F., Gelissen, J., \& van Oorschot, W. (2013). The multidimensionality of welfare state attitudes: A European cross-national study. Social Indicators Research, 113, 235-255.

Rueda, D., \& Thewissen, S. (2018). Automated but compensated? Technological change and redistribution in advanced democracies. In E. Paus (Hrsg.), Confronting dystopia (S. 134-155). Ithaca: Cornell University Press.

Rydgren, J. (Hrsg.). (2013). Class politics and the radical right. London: Routledge.

Svallfors, S. (1997). Worlds of welfare and attitudes to redistribution: A comparison of eight Western nations. European Sociological Review, 13, 283-304.

Svallfors, S. (Hrsg.). (2012). Contested welfare states: Welfare attitudes in Europe and beyond. Stanford: Stanford University Press.

Tawfik, A. (2014). Oesch class scheme. Create 16-class schema, 8-class schema and 5-class schema, ESS round 6. https://github.com/jolyphil/oesch-class. Zugegriffen: Juni 2020.

Tranter, B., \& Booth, K. (2015). Scepticism in a changing climate: A cross-national study. Global Environmental Change, 33, 154-164.

Vereinte Nationen (2020). Standard country or area codes for statistical use. https://unstats.un.org/unsd/ methodology/m49/. Zugegriffen: Nov. 2020.

van der Waal, J., Achterberg, P., Houtman, D., de Koster, W., \& Manevska, K. (2010). „Some are more equal than others": Economic egalitarianism and welfare chauvinism in the Netherlands. Journal of European Social Policy, 20, 350-363.

Zürn, M. (2020). Zurück zur Sozialistischen Internationale? Replik auf Carsten Nickel, Floris Biskamp und Michael Hartmann. Leviathan, 48, 161-175.

Zürn, M., \& de Wilde, P. (2016). Debating globalization: Cosmopolitanism and communitarianism as political ideologies. Journal of Political Ideologies, 21, 280-301. 
Thomas Lux geb. 1979. Dr., wissenschaftlicher Mitarbeiter am Lehrbereich Makrosoziologie der Humboldt-Universität zu Berlin. Forschungsschwerpunkte: Sozialstrukturanalyse, Lebenslaufsoziologie, Alter(n) und Generationen, Einstellungsforschung, politische Soziologie. Ausgewählte Veröffentlichungen: (mit S. Mau und F. Gülzau) Die drei Arenen der neuen Ungleichheitskonflikte. Eine sozialstrukturelle Positionsbestimmung der Einstellungen zu Umverteilung, Migration und sexueller Diversität, in: Berliner Journal für Soziologie, 2020; (mit J. O. Heuer, S. Mau, K. Zimmermann) Legitimizing inequality. The moral repertoires of meritocracy in four countries, in: Comparative Sociology, 2020; Die AfD und die unteren Statuslagen. Eine Forschungsnotiz zu Holger Lengfelds Studie „Die ,Alternative für Deutschland“: eine Partei für Modernisierungsverlierer?“, in: Kölner Zeitschrift für Soziologie und Sozialpsychologie, 2018.

Steffen Mau geb. 1968. Dr. rer. pol., Professor für Makrosoziologie an der Humboldt-Universität zu Berlin. Forschungsschwerpunkte: Soziale Ungleichheit, Grenzforschung, vergleichende Wohlfahrtsstaatsforschung, Sozialstrukturanalyse. Ausgewählte Veröffentlichungen: Sortiermaschinen. Die Neuerfindung der Grenze im 21. Jahrhundert, 2021; Lütten Klein. Leben in der ostdeutschen Transformationsgesellschaft, 2019; The metric society. On the quantification of the social world, 2019; Inequality, marketization and the majority class. Why did the European middle classes accept neoliberalism?, 2015.

Aljoscha Jacobi geb. 1990. M.Sc., wissenschaftlicher Mitarbeiter am Lehrbereich Makrosoziologie der Humboldt-Universität zu Berlin. Forschungsschwerpunkte: Politische Soziologie, Wohlfahrtsstaatsforschung, Arbeitsbeziehungen und Arbeitszeitregelungen. 